TRANSACTIONS OF THE

AMERICAN MATHEMATICAL SOCIETY

Volume 348, Number 5, May 1996

\title{
HIGHER LEFSCHETZ TRACES AND SPHERICAL EULER CHARACTERISTICS
}

\author{
ROSS GEOGHEGAN, ANDREW NICAS AND JOHN OPREA
}

\begin{abstract}
Higher analogs of the Euler characteristic and Lefschetz number are introduced. It is shown that they possess a variety of properties generalizing known features of those classical invariants. Applications are then given. In particular, it is shown that the higher Euler characteristics are obstructions to homotopy properties such as the TNCZ condition, and to a manifold being homologically Kähler.
\end{abstract}

The Lefschetz number of a self-map $f: X \rightarrow X$ of a space $X$ with finitely generated homology,

$$
L(f)=\sum_{k \geq 0}(-1)^{k} \operatorname{trace}\left(f_{k}: H_{k}(X ; \mathbb{Z}) / \text { torsion } \rightarrow H_{k}(X ; \mathbb{Z}) / \text { torsion }\right) \in \mathbb{Z},
$$

is a useful and well known invariant in algebraic topology. In case $f \equiv$ id, the identity map of $X, L(\mathrm{id})=\chi(X)=\sum_{k>0}(-1)^{k} \operatorname{rank} H_{k}(X ; \mathbb{Z})$ is the Euler characteristic of $X$. When $X$ is a finite complex, the Lefschetz number has a geometric interpretation in terms of fixed point theory; it is a "homological count" of the fixed points of $f$. One consequence is the celebrated Lefschetz Fixed Point Theorem which asserts that if $L(f) \neq 0$ then $f$ has a fixed point.

In this paper we study "higher" analogs of the Lefschetz number and the Euler characteristic. Let $\mathbb{F}$ be a field of coefficients and let $X$ be a space with finite dimensional homology over $\mathbb{F}$. Given a map $F: Y \times X \rightarrow X$, where $Y$ is an arbitrary parameter space, define a degree 0 homomorphism $L(F): H_{*}(Y ; \mathbb{F}) \rightarrow H_{*}(X ; \mathbb{F})$ by:

$$
L(F)(u)=\sum_{k \geq 0}(-1)^{k+n} \sum_{j=1}^{\beta_{k}} \bar{b}_{j}^{k} \cap F_{*}\left(u \times b_{j}^{k}\right)
$$

where $u \in H_{n}(Y ; \mathbb{F})$ and for each $k \geq 0\left\{b_{j}^{k} \mid j=1, \ldots, \beta_{k}\right\}$ is a basis for $H_{k}(X ; \mathbb{F})$ with corresponding dual basis $\left\{\bar{b}_{j}^{k} \mid j=1, \ldots, \beta_{k}\right\}$ for $H^{k}(X ; \mathbb{F})$. Here, we use Spanier's sign conventions [Sp] for the cap product and homology cross product. ${ }^{1}$

Received by the editors October 27, 1994.

1991 Mathematics Subject Classification. Primary 55M20; Secondary 55N45, 55R12, 58 F05.

The first author was partially supported by the National Science Foundation.

The second author was partially supported by the Natural Sciences and Engineering Research Council of Canada.

${ }^{1}$ Spanier's sign conventions for products, [Sp], differ from those of Dold, [Do3]. In particular, writing $\cap^{\prime}$ and $\cup^{\prime}$ for the cap and cup products of [Do3], we have $x \cap y=(-1)^{|x|(|x|-|y|)} x \cap^{\prime} y$ and $u \cup v=(-1)^{|u||v|} u \cup^{\prime} v$ where "| |" denotes the degree of a homology or cohomology class. 
This definition of $L(F)$ (up to sign conventions and placement of the parameter space on the left) is due to R. J. Knill, $[\mathrm{Kn}]$, so we call it the Knill trace. When $Y=S^{n}$ with $n>0$, the $n$-sphere, we can evaluate $L(F)$ on the fundamental homology class of $S^{n},\left[S^{n}\right] \in H_{n}\left(S^{n} ; \mathbb{F}\right)$, to obtain $L(F)\left(\left[S^{n}\right]\right) \in H_{n}(X ; \mathbb{F})$. This yields a map $\Lambda_{n}(f ; \mathbb{F}): \pi_{n}\left(X^{X}, f\right) \rightarrow H_{n}(X ; \mathbb{F})$ which we call the $n^{\text {th }}$ spherical Lefschetz characteristic based at $f: X \rightarrow X$ (see Definition 5.1), where $\pi_{n}\left(X^{X}, f\right)$ is the $n^{\text {th }}$ homotopy group of the function space $X^{X}$ of self-maps of $X$ and $f \in X^{X}$ is a basepoint. In case $f=\operatorname{id}_{X}$, the identity map of $X$, we write $\chi_{n}(X ; \mathbb{F}) \equiv \Lambda_{n}\left(\operatorname{id}_{X} ; \mathbb{F}\right)$ and call $\chi_{n}(X ; \mathbb{F})$ the $n^{\text {th }}$ spherical Euler characteristic of $X$. Our definitions of $\Lambda_{n}(f ; \mathbb{F})$ and $\chi_{n}(X ; \mathbb{F})$ also make sense in case $n=0$ with $\left[S^{0}\right] \in H_{0}\left(S^{0} ; \mathbb{F}\right)$ chosen appropriately. The pointed set $\pi_{0}\left(X^{X}, f\right)$ is the set of homotopy classes of maps $g: X \rightarrow X$ with $f$ representing the distinguished basepoint. For $X$ path connected, $\Lambda_{0}(f ; \mathbb{F})([g])=L(g)\left[x_{0}\right]$ where $L(g)$ is the Lefschetz number of $g: X \rightarrow X$ and $\left[x_{0}\right]$ is the zero-dimensional homology class represented by a basepoint $x_{0} \in X$; in particular, $\chi_{0}(X ; \mathbb{F})([g])=L(g)\left[x_{0}\right]$.

In $[\mathrm{GN}]$, the invariant $\chi_{1}(X ; \mathbb{F})$ was studied extensively, with applications to group theory, and its analogy to the classical Euler characteristic was elaborated.

When $X$ is a finite complex, $L(F)$ is closely related to the fixed point theory of $F: Y \times X \rightarrow X$. The fixed point set of $F$ is the set

$$
\operatorname{Fix}(F)=\{(y, x) \in Y \times X \mid F(y, x)=x\} .
$$

Suppose $X \subset \mathbb{R}^{m}$ is a compact polyhedron, $M$ is a regular neighborhood of $X$ and $r: M \rightarrow X$ a strong deformation retraction. Let $F^{\prime}: Y \times M \rightarrow M$ be $F^{\prime}=$ $F \circ\left(\operatorname{id}_{Y} \times r\right)$. Clearly, $\operatorname{Fix}\left(F^{\prime}\right)=\operatorname{Fix}(F)$ and $F^{\prime}$ has no fixed points on $Y \times \partial M$. Let $[M] \in H_{m}(M, \partial M ; \mathbb{F})$ be the fundamental class of $M$ and let $\left[\mathbb{R}^{m}\right]$ be the generator of $H_{m}\left(\mathbb{R}^{m}, \mathbb{R}^{m}-\{0\} ; \mathbb{F}\right)$ determined by the standard orientation of $\mathbb{R}^{m}$. Define

$$
\Phi:(Y \times M, Y \times M-\operatorname{Fix}(F)) \rightarrow M \times\left(\mathbb{R}^{m}, \mathbb{R}^{m}-\{0\}\right)
$$

by $\Phi=\left(F^{\prime}, i \circ p-i \circ F^{\prime}\right)$ where $p: Y \times M \rightarrow M$ is projection and $i: M \hookrightarrow \mathbb{R}^{m}$ is inclusion. Following $[\mathrm{Le}]$ and $\left[\mathrm{D}_{1}\right]$, Knill $[\mathrm{Kn}]$ defines the intersection class of $F$ to be the degree 0 homomorphism $I(F): H_{*}(Y ; \mathbb{F}) \rightarrow H_{*}(X ; \mathbb{F})$ given in degree $n$ by the composite:

$$
\begin{aligned}
H_{n}(Y ; \mathbb{F}) & \stackrel{\times[M]}{\longrightarrow} H_{n+m}(Y \times(M, \partial M) ; \mathbb{F}) \rightarrow H_{n+m}(Y \times M, Y \times M-\operatorname{Fix}(F) ; \mathbb{F}) \\
& \stackrel{\Phi_{*}}{\longrightarrow} H_{n+m}\left(M \times\left(\mathbb{R}^{m}, \mathbb{R}^{m}-\{0\}\right) ; \mathbb{F}\right) \stackrel{\cong}{\longrightarrow} H_{n}(M ; \mathbb{F}) \stackrel{r_{*}}{\longrightarrow} H_{n}(X ; \mathbb{F})
\end{aligned}
$$

where $H_{n+m}\left(M \times\left(\mathbb{R}^{m}, \mathbb{R}^{m}-\{0\}\right) ; \mathbb{F}\right) \stackrel{\cong}{\rightrightarrows} H_{n}(M ; \mathbb{F})$ is the inverse of the isomorphism

$$
H_{n}(M ; \mathbb{F}) \stackrel{\cong}{\rightrightarrows} H_{n+m}\left(M \times\left(\mathbb{R}^{m}, \mathbb{R}^{m}-\{0\}\right) ; \mathbb{F}\right), \quad \beta \mapsto \beta \times\left[\mathbb{R}^{m}\right] .
$$

Let $S: H_{*}(Y ; \mathbb{F}) \rightarrow H_{*}(Y ; \mathbb{F})$ be the isomorphism $S(\beta)=(-1)^{n} \beta$ for $\beta \in H_{n}(Y ; \mathbb{F})$. Knill shows [Kn, Theorem 1]:

$$
I(F)=L(F) \circ S .
$$

In particular, if $L(F) \neq 0$ then $I(F) \neq 0$ and consequently $\operatorname{Fix}(F) \neq \emptyset$. 
The homomorphism $I(F)$ also has a description in terms of the transfer maps of [BG], [D $\mathrm{D}_{2}$ ]. Let $\bar{F}: Y \times X \rightarrow Y \times X$ be $\bar{F}(y, x)=(y, F(y, x))$ where $X$ is a compact polyhedron. Consider the trivial fibration $X \rightarrow Y \times X \stackrel{p}{\rightarrow} Y$. Since $\bar{F}$ is a fiber map, i.e. $p=p \circ \bar{F}$, there is an $S-$ map $\Sigma^{\infty} Y_{+} \stackrel{\tau(\bar{F})}{\longrightarrow} \Sigma^{\infty}(Y \times X)_{+}$. Here, the subscript "+" indicates union with a disjoint basepoint and " $\Sigma^{\infty}$ " denotes the suspension spectrum of a space. The $S$-map $\tau(\bar{F})$ induces a homomorphism in homology $\tau(\bar{F})_{*}: H_{*}(Y ; \mathbb{F}) \rightarrow H_{*}(Y \times X ; \mathbb{F})$. Composition of $\tau(\bar{F})_{*}$ with the homomorphism $p_{*}: H_{*}(Y \times X ; \mathbb{F}) \rightarrow H_{*}(X ; \mathbb{F})$ induced by projection $p: Y \times X \rightarrow X$ is $I(F)$; see $\left[\mathrm{D}_{2},(3.3)\right]$ and $[\mathrm{BG}, \S 9]$.

The first part of this paper, $\S \S 1-5$, consists of generalizations to the Knill trace, $L(F)$, (and, in particular, to our spherical Lefschetz and Euler characteristics) of familiar properties of the classical Lefschetz number and Euler characteristic. We prove a "higher" algebraic analog of the Hopf trace formula (Theorem 3.1) and apply it to give a chain level formula for the Knill trace when $X$ is a finite simplicial complex (Proposition 3.4). Theorem 4.1 is a "Sum Theorem" for $L(F)$ generalizing the formula $\chi(X)=\chi(A)+\chi(X, A)$ for a topological pair $(X, A)$. Theorem 4.3 is a "Product Theorem" for $L(F)$ in case $X$ is the total space of a Serre fibration $K \rightarrow X \rightarrow B$ where $F: Y \times X \rightarrow X$ is fiber preserving, $B$ is a finite complex and $K$ has finite dimensional homology over $\mathbb{F}$. This generalizes the formula $\chi(X)=\chi(K) \chi(B)$. Another kind of product formula is given in Theorem 4.5.

The remainder of the paper is concerned with some applications. Let $E: X^{X} \times$ $X \rightarrow X$ be the evaluation map, let $h: \pi_{n}\left(X^{X}, f\right) \rightarrow H_{n}\left(X^{X} ; \mathbb{F}\right)$ be the Hurewicz map over $\mathbb{F}$, and let $H_{n}\left(X^{X} ; \mathbb{F}\right) \otimes H^{q}\left(X^{X} \times X ; \mathbb{F}\right) \stackrel{\backslash}{\rightarrow} H^{q-n}(X ; \mathbb{F})$ be the slant product. For $\hat{\alpha} \in \pi_{n}\left(X^{X}, f\right)$ define a derivation $\lambda_{\hat{\alpha}}: H^{*}(X ; \mathbb{F}) \rightarrow H^{*-n}(X ; \mathbb{F})$ by the formula $\lambda_{\hat{\alpha}}(x)=h(\hat{\alpha}) \backslash E^{*}(x)$. Given a basepoint $x_{0} \in X$, let $\alpha_{\bullet}=\operatorname{ev}_{\#}(\hat{\alpha}) \in$ $\pi_{n}\left(X, f\left(x_{0}\right)\right)$ where ev: $X^{X} \rightarrow X$ is evaluation at $x_{0}$. For each $k \geq 0$, let $\left\{b_{j}^{k}\right\}$ be a basis for $H_{k}(X ; \mathbb{F})$ with corresponding dual basis $\left\{\bar{b}_{j}^{k}\right\}$ for $H^{k}(X ; \mathbb{F})$.

Proposition 6.8. If $n>0$ then $\Lambda_{n}(f ; \mathbb{F})(\hat{\alpha})=\sum_{k \geq 0}(-1)^{k} \sum_{j} \lambda_{\hat{\alpha}}\left(\bar{b}_{j}^{k}\right) \cap b_{j}^{k}$.

The definition of $\Lambda_{n}(f ; \mathbb{F})$ leads to a different formula for $\Lambda_{n}(f ; \mathbb{F})$ in terms of $\lambda_{\hat{\alpha}}$ (Proposition 6.9). Comparison of the two yields an interesting formula which does not involve our new invariants:

Theorem 6.10. For $X$ path connected and $n>0$,

$$
L(f) h\left(\alpha_{\bullet}\right)=\left(\mathrm{id}-f_{*}\right)\left(\sum_{k \geq 0}(-1)^{k+n} \sum_{j} \lambda_{\hat{\alpha}}\left(\bar{b}_{j}^{k}\right) \cap b_{j}^{k}\right) .
$$

In particular, this implies:

Corollary 6.12. If $n>0, f_{*}: H_{n}(X ; \mathbb{F}) \rightarrow H_{n}(X ; \mathbb{F})$ is the identity and $L(f) \neq 0$ $\bmod p$, where $p \geq 0$ is the characteristic of $\mathbb{F}$, then the composite

$$
\pi_{n}\left(X^{X}, f\right) \stackrel{\mathrm{ev}_{\#}}{\longrightarrow} \pi_{n}\left(X, f\left(x_{0}\right)\right) \stackrel{h}{\longrightarrow} H_{n}(X ; \mathbb{F})
$$

is zero.

The special case of Corollary 6.12 in which $f=\mathrm{id}$, so that $L(f)=\chi(X)$, was proved in $\left[\mathrm{Go}_{2}\right]$. 
When $f=\mathrm{id}$, the derivation $\lambda_{\hat{\alpha}}$ is more familiar. In that case $\hat{\alpha} \in \pi_{n}\left(X^{X}, \mathrm{id}_{X}\right)$, where $n>0$, may be viewed as a clutching map along the equator of $S^{n+1}$ which constructs a "Wang fibration" $X \stackrel{i}{\rightarrow} E \rightarrow S^{n+1}$ (see $\S 6$ ). Such a fibration has a long exact Wang sequence associated to it:

$$
\cdots \rightarrow H^{q}(E ; \mathbb{F}) \stackrel{i^{*}}{\rightarrow} H^{q}(X ; \mathbb{F}) \stackrel{\lambda}{\rightarrow} H^{q-n}(X ; \mathbb{F}) \rightarrow H^{q+1}(E ; \mathbb{F}) \rightarrow \cdots
$$

and the homomorphism $\lambda$ appearing in this sequence coincides with $\lambda_{\hat{\alpha}}$ above. The exactness of the Wang sequence tells us that $\lambda_{\hat{\alpha}}=0$ if and only if the fibration $X \rightarrow E \rightarrow S^{n+1}$ is totally non-cohomologous to zero (see $\S 6$ ) and so our formula for $\chi_{n}(X ; \mathbb{F})$ in terms of $\lambda_{\hat{\alpha}}$ (Proposition 6.8) permits us to conclude:

Theorem 7.3. Let $\hat{\alpha} \in \pi_{n}\left(X^{X}, \mathrm{id}_{X}\right)$ where $n>0$. If the associated Wang fibration $X \rightarrow E \rightarrow S^{n+1}$ is totally noncohomologous to zero, then $\chi_{n}(X ; \mathbb{F})(\hat{\alpha})=0$.

Using this, we can show that spherical Euler characteristics are obstructions to interesting geometric properties of manifolds. Leaving definitions and details until $\S 7$, we simply state:

Corollary 7.5. If $X$ is a closed symplectic manifold and $\hat{\alpha} \in \pi_{1}\left(X^{X}, \mathrm{id}_{X}\right)$ corresponds to a Hamiltonian $S^{1}$-action, then $\chi_{1}(X ; \mathbb{Q})(\hat{\alpha})=0 .^{2}$

Proposition 7.7. If $X$ is homologically Kähler and simply connected then

$$
\chi_{n}(X ; \mathbb{Q})(\hat{\alpha})=0 \text { for all } \hat{\alpha} \in \pi_{n}\left(X^{X}, \operatorname{id}_{X}\right) \text { and } n \geq 1 .
$$

For example, this proposition applies to the complex manifold $\mathbb{C} P^{n}$ which is Kähler and simply connected.

Conventions and Notation. Throughout this paper we use singular homology and cohomology; furthermore, all homology and cohomology groups will have coefficients in a field $\mathbb{F}$ unless otherwise indicated, i.e. the groups $H_{*}(X)$ and $H^{*}(X)$ are understood to have coefficients in $\mathbb{F}, H_{*}(X ; R)$ and $H^{*}(X ; R)$ indicate coefficients in a commutative ring $R$. We use Spanier's sign conventions [Sp] for cross, cup, cap and slant products. We work in a suitable category of spaces so that the "exponential rule" identifying the space of continuous maps $Y \times X \rightarrow X$ with the space of continuous maps $Y \rightarrow X^{X}$ is valid. Typically in this paper, $X$ will be a space whose homology $H_{*}(X)$ is finite dimensional over $\mathbb{F}$; in particular, we usually do not require $X$ to be a finite complex.

Acknowledgment. We thank Albrecht Dold for some very helpful comments.

\section{TRACES}

Let $V_{*}$ be a finite dimensional graded vector space over a field $\mathbb{F}$. The dual of $V_{*}$ is the graded vector space, $V^{*}$, given by $V^{i}=\operatorname{Hom}_{\mathbb{F}}\left(V_{i}, \mathbb{F}\right)$. Let $C_{*}$ be another (not necessarily finite dimensional) graded vector space over $\mathbb{F}$. Suppose we are given a cap product with values in $C_{*}$, i.e. a collection of homomorphisms $V^{r} \otimes V_{s} \stackrel{\cap}{\longrightarrow}$ $C_{s-r}$. Let $\langle\cdot, \cdot\rangle: V^{i} \otimes V_{i} \rightarrow \mathbb{F}$ denote the natural dual pairing. There is a natural isomorphism $\Psi: V^{k} \otimes V_{k+n} \rightarrow \operatorname{Hom}_{\mathbb{F}}\left(V_{k}, V_{k+n}\right)$ given by $\Psi(g \otimes x)(y)=\langle g, y\rangle x$ for $x \in V_{k+n}, g \in V^{k}$ and $y \in V_{k}$.

\footnotetext{
${ }^{2}$ See note added in proof.
} 
Definition 1.1. The trace of a homomorphism $f \in \operatorname{Hom}_{\mathbb{F}}\left(V_{k}, V_{k+n}\right)$ is the vector:

$$
\operatorname{trace}(f ; \cap) \equiv \cap \circ \Psi^{-1}(f) \in C_{n} .
$$

As the notation suggests, trace $(f ; \cap)$ depends on the cap product as well as on $f$.

Proposition 1.2. Let $\left\{b_{1}^{k}, \ldots, b_{m_{k}}^{k}\right\}$ be a basis for $V_{k}$ and $\left\{\bar{b}_{1}^{k}, \ldots, \bar{b}_{m_{k}}^{k}\right\}$ the corresponding dual basis for $V^{k}$. Then trace $(f ; \cap)=\sum_{j=1}^{m_{k}} \bar{b}_{j}^{k} \cap f\left(b_{j}^{k}\right)$.

Proof. Let $\left\{b_{1}^{k+n}, \ldots, b_{m_{k+n}}^{k+n}\right\}$ be a basis for $V_{k+n}$. We can write

$$
f=\sum_{i, j} f_{i j} \Psi\left(\bar{b}_{j}^{k} \otimes b_{i}^{k+n}\right)
$$

where $f_{i j} \in \mathbb{F}$. It follows that $f\left(b_{j}^{k}\right)=\sum_{i} f_{i j} b_{i}^{k+n}$. Then

$$
\begin{aligned}
\operatorname{trace}(f ; \cap) & =\cap \circ \Psi^{-1}(f)=\sum_{i j} f_{i j} \bar{b}_{j}^{k} \cap b_{i}^{k+n} \\
& =\sum_{j} \bar{b}_{j}^{k} \cap\left(\sum_{i} f_{i j} b_{i}^{k+n}\right)=\sum_{j} \bar{b}_{j}^{k} \cap f\left(b_{j}^{k}\right) .
\end{aligned}
$$

Definition 1.3. Let $V_{*}$ be a finite dimensional graded vector space equipped with a cap product taking values in the graded vector space $C_{*}$. Let $\theta: V_{*} \rightarrow V_{*}$ a be homomorphism of degree $n$. Then the Lefschetz class of $\theta, L_{n}(\theta) \in C_{n}$, is the alternating sum of traces:

$$
L_{n}(\theta)=\sum_{k}(-1)^{k+n} \operatorname{trace}\left(\theta_{k} ; \cap\right)
$$

Remark 1.4. Suppose that there is $c_{0} \in C_{0}$ such that $x \cap y=\langle x, y\rangle c_{0}$ for all $k$, $x \in V^{k}$ and $y \in V_{k}$. Then, in this case, for a degree 0 homomorphism $\theta: V_{*} \rightarrow V_{*}$ we have $\operatorname{trace}\left(\theta_{k} ; \cap\right)=\operatorname{trace}\left(\theta_{k}\right) c_{0}$ and so $L_{0}(\theta)=L(\theta) c_{0}$ where $L(\theta)$ is the classical Lefschetz number.

There is an obvious "dual" formulation of the preceding theory which we now outline.

There is natural isomorphism $\bar{\Psi}: V^{k-n} \otimes V_{k} \rightarrow \operatorname{Hom}_{\mathbb{F}}\left(V^{k}, V^{k-n}\right)$ given by $\bar{\Psi}(g \otimes x)(y)=\langle y, x\rangle g$ for $g \in V^{k-n}, x \in V_{k}$ and $y \in V^{k}$.

Definition 1.5. The trace of a homomorphism $f \in \operatorname{Hom}_{\mathbb{F}}\left(V^{k}, V^{k-n}\right)$ is the vector:

$$
\operatorname{trace}(f ; \cap) \equiv \cap \circ \bar{\Psi}^{-1}(f) \in C_{n} .
$$

Just as in Proposition 1.2, it is easy to show:

Proposition 1.6. Let $\left\{b_{1}^{k}, \ldots, b_{m_{k}}^{k}\right\}$ be a basis for $V_{k}$ and $\left\{\bar{b}_{1}^{k}, \ldots, \bar{b}_{m_{k}}^{k}\right\}$ the corresponding dual basis for $V^{k}$. Then trace $(f ; \cap)=\sum_{j=1}^{m_{k}} f\left(\bar{b}_{j}^{k}\right) \cap b_{j}^{k}$.

Definition 1.7. Let $V_{*}$ be a finite dimensional graded vector space equipped with a cap product taking values in the graded vector space $C_{*}$. Let $\theta: V^{*} \rightarrow V^{*}$ a be homomorphism of degree $-n$. Then the Lefschetz class of $\theta, L_{n}(\theta) \in C_{n}$, is the alternating sum of traces: $L_{n}(\theta)=\sum_{k}(-1)^{k+n} \operatorname{trace}\left(\theta^{k} ; \cap\right)$. 


\section{Traces and Parametrized Maps}

Let $(X, A)$ be a topological pair such that $H_{*}(X, A)$ is finite dimensional over $\mathbb{F}$. Given a continuous map $F: Y \times(X, A) \rightarrow(X, A)$ of pairs, where $Y$ is a parameter space, and $u \in H_{n}(Y)$, let $F_{u}: H_{*}(X, A) \rightarrow H_{*}(X, A)$ be the degree $n$ homomorphism given by $F_{u}(x)=F_{*}(u \times x)$. We use the cap product $H^{j}(X, A) \otimes H_{j+n}(X, A) \stackrel{\cap}{\longrightarrow} H_{n}(X)$ to define the Lefschetz classes $L_{n}\left(F_{u}\right) \in H_{n}(X)$ (see Definition 1.3).

The following definition of $L(F)$, up to sign conventions and placement of the parameter space on the left, is essentially due to R. J. Knill, [Kn].

Definition 2.1. The Knill trace of $F: Y \times(X, A) \rightarrow(X, A)$ is the degree 0 homomorphism $L(F): H_{*}(Y) \rightarrow H_{*}(X)$ given by $L(F)(u)=L_{n}\left(F_{u}\right) \in H_{n}(X)$ where $u \in H_{n}(Y)$.

Clearly, $L(F)$ depends only on the homotopy class of $F$ as a map of pairs.

By Proposition 1.2,

$$
L(F)(u)=\sum_{k \geq 0}(-1)^{k+n} \sum_{j=1}^{\beta_{k}} \bar{b}_{j}^{k} \cap F_{*}\left(u \times b_{j}^{k}\right)
$$

where $u \in H_{n}(Y)$ and for each $k \geq 0\left\{b_{j}^{k} \mid j=1, \ldots, \beta_{k}\right\}$ is a basis for $H_{k}(X, A)$ with corresponding dual basis $\left\{\bar{b}_{j}^{k} \mid j=1, \ldots, \beta_{k}\right\}$ for $H^{k}(X, A)$.

Remark 2.2. Let $y_{0} \in Y$. Define $f:(X, A) \rightarrow(X, A)$ by $f=F\left(y_{0}, \cdot\right)$. Suppose $X$ is path connected and $x_{0} \in X$. Then, by Remark $1.4, L(F)\left(\left[y_{0}\right]\right)=L(f)\left[x_{0}\right]$ where $L(f)$ is the classical Lefschetz number of $f$ and $\left[y_{0}\right] \in H_{0}(Y)$ and $\left[x_{0}\right] \in H_{0}(X)$ have the obvious meaning.

Example 2.3. The Knill trace may be calculated quite easily for the multiplication map $\mu: G \times G \rightarrow G$ of a compact Lie group in the case where $\mathbb{F}$ is of characteristic zero and the cohomology and homology are dual exterior algebras (e.g. $U(n), S p(n), S U(n)$ [W, pp. 343-345]). Taking all cohomology and homology with coefficients in $\mathbb{F}$, we write

$$
H^{*}(G)=\Lambda\left(\bar{x}_{1}, \ldots, \bar{x}_{n}\right), \quad H_{*}(G)=\Lambda\left(x_{1}, \ldots, x_{n}\right)
$$

where $\bar{x}_{i}$ is Kronecker dual to $x_{i}$. The monomials $x_{I}=x_{i_{1}} x_{i_{2}} \cdots x_{i_{k}}$ with $i_{1}<i_{2}<$ $\cdots<i_{k}$ form an additive basis for homology and similarly for $\bar{x}_{I}$ in cohomology. Now,

$$
\bar{x}_{J} \cap \mu_{*}\left(x_{I} \times x_{J}\right)= \begin{cases}0 & \text { for } I \cap J \neq \emptyset, \\ x_{I} & \text { for } I \cap J=\emptyset\end{cases}
$$

since $\mu_{*}\left(x_{I} \times x_{J}\right)=x_{I} \wedge x_{J}$ is the exterior product. (The cap product may be computed by Kronecker duality as in [W, p. 343].) Therefore, for a monomial $x_{I}$, the only nonzero terms in $L(\mu)\left(x_{I}\right)$ occur for monomials $x_{J}$ with $J$ disjoint from $I$. These $x_{J}$ 's come from a sub-exterior algebra $\bar{\Lambda}=\Lambda\left(x_{j_{1}}, \ldots, x_{j_{l}}\right)$ generated by the $x_{j}$ which do not appear in $x_{I}$. Thus, for $x_{I} \neq 1$ or $z$ (where $z$ is the top class 
$\left.x_{1} \wedge \cdots \wedge x_{n}\right)$, we have

$$
\begin{aligned}
L(\mu)\left(x_{I}\right) & =\sum_{k \geq 0}(-1)^{k+|I|} \sum_{|J|=k} x_{J} \cap \mu_{*}\left(x_{I} \times x_{J}\right) \\
& =(-1)^{|I|} \sum_{k \geq 0}(-1)^{k} x_{I} \cdot \operatorname{dim} \bar{\Lambda}_{k} \\
& =(-1)^{|I|} x_{I} \cdot \chi(\bar{\Lambda})
\end{aligned}
$$

where $\chi(\bar{\Lambda})=\sum_{k>0}(-1)^{k} \operatorname{dim} \bar{\Lambda}_{k}$ is the Euler characteristic of the exterior algebra $\bar{\Lambda}$. But, it is well known and easy to see that this Euler characteristic is zero. Hence, $L(\mu)\left(x_{I}\right)=0$.

If $x_{I}=1 \in H_{0}(G)$, then clearly $L(\mu)(1)=\chi(G)=0$ as well. Since $L(\mu)$ is a homomorphism, only the case $x_{I}=z$ remains. For dimensional reasons, $\mu_{*}\left(z \times x_{J}\right)=0$ for all $x_{J}$ except $x_{J}=1$. In that case $1 \cap \mu_{*}(z \times 1)=z$. Note that $z=x_{1} \wedge \cdots \wedge x_{n}$, so $|J|=n=\operatorname{dim} G$. Thus, for $y \in H_{m}(G)$,

$$
L(\mu)(y)= \begin{cases}0 & \text { for } m<\operatorname{dim} G \\ (-1)^{\operatorname{dim} G} y & \text { for } m=\operatorname{dim} G\end{cases}
$$

This example generalizes [GN, Example 3.1] where it was shown that the spherical Euler characteristic of the generator $U \in H_{1}\left(S^{1}\right)$ is $-U$.

Example 2.4. Knill traces of maps $F: Y \times S^{N} \rightarrow S^{N}$ also lend themselves to calculation. For instance, consider the action $A: S^{3} \times S^{2} \rightarrow S^{2}$ given by regarding $S^{2}$ as the homogeneous space $S^{3} / S^{1}$ arising from the Hopf principal bundle $S^{1} \rightarrow$ $S^{3} \rightarrow S^{2}$. As usual $L(A)(1)=\chi\left(S^{2}\right)=2$ for $1 \in H_{0}\left(S^{3}\right)$. If $U \in H_{3}\left(S^{3}\right)$, however, $L(A)(U)=0$ for dimensional reasons. Hence, the Knill trace is only nontrivial in degree 0 .

Example 2.5. Similarly, the principal bundle $S U(n) \rightarrow S U(n+1) \rightarrow S^{2 n+1}$ induces an action $A: S U(n+1) \times S^{2 n+1} \rightarrow S^{2 n+1}$. Now, over a field of characteristic zero, the map on homology $A \mid: H_{*}(S U(n+1)) \rightarrow H_{*}\left(S^{2 n+1}\right)$ is $\Lambda\left(x_{3}, x_{5}, \ldots, x_{2 n+1}\right)$ $\rightarrow \Lambda(U), x_{i} \mapsto 0$ for $i<2 n+1$ and $x_{2 n+1} \mapsto U$. For these reasons, as well as dimensional considerations, it is easy to see that

$$
L(A)\left(x_{I}\right)= \begin{cases}0 & \text { for } I \neq\{2 n+1\} \\ -U & \text { for } I=\{2 n+1\}\end{cases}
$$

We can make a similar analysis for the $U(n+1)$ action on $S^{2 n+1}$, but this will be a consequence of the following naturality property of the Knill trace:

Theorem 2.6 (Naturality). Let $F: Y \times(X, A) \rightarrow(X, A), F^{\prime}: Y^{\prime} \times\left(X^{\prime}, A^{\prime}\right) \rightarrow$ $\left(X^{\prime}, A\right), g: Y \rightarrow Y^{\prime}$ and $h:(X, A) \rightarrow\left(X^{\prime}, A^{\prime}\right)$ be maps such that the diagram

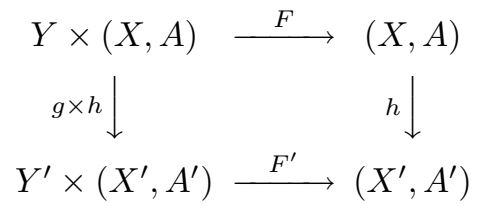


induces a commutative diagram in relative homology. Suppose $h$ induces an isomorphism in relative homology. Then for $y \in H_{*}(Y)$,

$$
h_{*}(L(F)(y))=L\left(F^{\prime}\right)\left(g_{*}(y)\right) .
$$

Proof. For $k \geq 0$, let $\left\{b_{j}^{k}\right\}$ be a basis for $H_{*}(X, A)$ with corresponding dual basis $\left\{\bar{b}_{j}^{k}\right\}$ for $H_{*}(X, A)$. Since $h_{*}$ is an isomorphism $\left\{h_{*}\left(b_{j}^{k}\right)\right\}$ is a basis for $H_{*}\left(X^{\prime}, A^{\prime}\right)$ with dual basis $\overline{h_{*}\left(b_{j}^{k}\right)}=\left(h^{*}\right)^{-1}\left(\bar{b}_{j}^{k}\right)$. By hypothesis, $F_{*}=\left(h_{*}\right)^{-1} F_{*}^{\prime}(g \times h)_{*}$ and so for $y \in H_{n}(Y)$

$$
\begin{aligned}
h_{*}\left(\bar{b}_{j}^{k} \cap F_{*}\left(y \times b_{j}^{k}\right)\right) & =h_{*}\left(\bar{b}_{j}^{k} \cap\left(h_{*}\right)^{-1} F_{*}^{\prime}(g \times h)_{*}\left(y \times b_{j}^{k}\right)\right) \\
& =h_{*}\left(\left(h_{*}\right)^{-1}\left(\left(h^{*}\right)^{-1}\left(\bar{b}_{j}^{k}\right) \cap F_{*}^{\prime}\left(g(y) \times h_{*}\left(b_{j}^{k}\right)\right)\right)\right) \\
& =\overline{h_{*}\left(b_{j}^{k}\right)} \cap F_{*}^{\prime}\left(g(y) \times h_{*}\left(b_{j}^{k}\right)\right) .
\end{aligned}
$$

Using this expression, we have:

$$
\begin{aligned}
h_{*}(L(F)(y)) & =h_{*}\left(\sum_{k \geq 0}(-1)^{k+n} \sum_{j} \bar{b}_{j}^{k} \cap F_{*}\left(y \times b_{j}^{k}\right)\right) \\
& =\sum_{k \geq 0}(-1)^{k+n} \sum_{j} h_{*}\left(\bar{b}_{j}^{k} \cap F_{*}\left(y \times b_{j}^{k}\right)\right) \\
& =\sum_{k \geq 0}(-1)^{k+n} \sum_{j} \overline{h_{*}\left(b_{j}^{k}\right)} \cap F_{*}^{\prime}\left(g(y) \times h_{*}\left(b_{j}^{k}\right)\right) \\
& =L\left(F^{\prime}\right)\left(g_{*}(y)\right) .
\end{aligned}
$$

Example 2.7. Suppose $K$ is a closed subgroup of the compact Lie group $G$ with $\operatorname{dim} K<\operatorname{dim} G$ and the map $\bar{\mu}: K \times G \rightarrow G$ is the restriction of $G$ 's multiplication. Since $L(\mu)$ is zero except for the top class $z \in H_{\operatorname{dim} G}(G)$ (see Example 2.3 above) and $\operatorname{dim} K<\operatorname{dim} G$, Theorem 2.6 implies $L(\bar{\mu})=0$ identically.

Let $E: X^{X} \times X \rightarrow X$ be the evaluation map, i.e. $E(f, x)=f(x)$.

Definition 2.8. The evaluation trace, $\mathcal{E}: H_{*}\left(X^{X}\right) \rightarrow H_{*}(X)$, is the degree 0 homomorphism given by the Knill trace of the evaluation map, i.e. $\mathcal{E} \equiv L(E)$.

The evaluation trace is the "universal example" of the Knill trace in the following sense. Given any map $F: Y \times X \rightarrow X$ with adjoint $\hat{F}: Y \rightarrow X^{X}$ defined by $\hat{F}(y)(x)=F(y, x)$ we can write $F=E \circ(\hat{F} \times$ id $)$ and so by Theorem 2.6:

Proposition 2.9. $L(F)=\mathcal{E} \circ \hat{F}_{*}$.

The evaluation trace can be expressed in terms of cohomology as follows. Define a degree 0 homomorphism $\bar{\lambda}: H_{*}\left(X^{X}\right) \rightarrow \operatorname{Hom}\left(H^{*}(X), H^{*}(X)\right)$ by $\bar{\lambda}(\beta)(z)=$ $\beta \backslash E^{*}(z)$ where $\beta \in H_{n}\left(X^{X}\right), z \in H^{k}(X)$ and $H_{n}\left(X^{X}\right) \otimes H^{k}\left(X^{X} \times X\right) \stackrel{\backslash}{\rightarrow} H^{k-n}(X)$ is the slant product (see [Sp, p. 351]). Observe that $\bar{\lambda}(\beta): H^{*}(X) \rightarrow H^{*}(X)$ has degree $-n$ so that we may apply Definition 1.7 to obtain $L_{n}(\bar{\lambda}(\beta)) \in H_{n}(X)$. 
Theorem 2.10. For $\beta \in H_{n}\left(X^{X}\right), \mathcal{E}(\beta)=(-1)^{n} L_{n}(\bar{\lambda}(\beta))$.

Proof. For $k \geq 0$, let $\left\{b_{j}^{k}\right\}$ be a basis for $H_{k}(X)$ and $\left\{\bar{b}_{j}^{k}\right\}$ the corresponding dual basis for $H^{k}(X)$. Let $\beta \in H_{n}\left(X^{X}\right)$. Then for any $z \in H^{k}(X)$

$$
\left\langle\beta \backslash E^{*}(z), b_{\ell}^{k-n}\right\rangle=\left\langle E^{*}(z), \beta \times b_{\ell}^{k-n}\right\rangle=\left\langle z, E_{*}\left(\beta \times b_{\ell}^{k-n}\right)\right\rangle
$$

and consequently

$$
\begin{aligned}
\bar{\lambda}(\beta)(z) & =\sum_{\ell}\left\langle\bar{\lambda}(\beta)(z), b_{\ell}^{k-n}\right\rangle \bar{b}_{\ell}^{k-n}=\sum_{\ell}\left\langle\beta \backslash E^{*}(z), b_{\ell}^{k-n}\right\rangle \bar{b}_{\ell}^{k-n} \\
& =\sum_{\ell}\left\langle z, E_{*}\left(\beta \times b_{\ell}^{k-n}\right)\right\rangle \bar{b}_{\ell}^{k-n} .
\end{aligned}
$$

Using this expression for $\bar{\lambda}(\beta)$, we obtain:

$$
\begin{aligned}
\sum_{j} \bar{\lambda}(\beta)\left(\bar{b}_{j}^{k}\right) \cap b_{j}^{k} & =\sum_{j}\left(\sum_{\ell}\left\langle\bar{b}_{j}^{k}, E_{*}\left(\beta \times b_{\ell}^{k-n}\right)\right\rangle \bar{b}_{\ell}^{k-n}\right) \cap b_{j}^{k} \\
& =\sum_{\ell} \bar{b}_{\ell}^{k-n} \cap\left(\sum_{j}\left\langle\bar{b}_{j}^{k}, E_{*}\left(\beta \times b_{\ell}^{k-n}\right)\right\rangle b_{j}^{k}\right) \\
& =\sum_{\ell} \bar{b}_{\ell}^{k-n} \cap E_{*}\left(\beta \times b_{\ell}^{k-n}\right) .
\end{aligned}
$$

Thus

$$
(-1)^{n} L_{n}(\bar{\lambda}(\beta))=\sum_{k \geq 0}(-1)^{k} \sum_{j} \bar{\lambda}(\beta)\left(\bar{b}_{j}^{k}\right) \cap b_{j}^{k}=\sum_{k \geq 0}(-1)^{k} \sum_{\ell} \bar{b}_{\ell}^{k-n} \cap E_{*}\left(\beta \times b_{\ell}^{k-n}\right) .
$$

Since $b_{\ell}^{k-n}=0$ if $k<n$, we can put $s=k-n$ yielding:

$$
\sum_{k \geq 0}(-1)^{k} \sum_{\ell} \bar{b}_{\ell}^{k-n} \cap E_{*}\left(\beta \times b_{\ell}^{k-n}\right)=\sum_{s \geq 0}(-1)^{s+n} \sum_{\ell} \bar{b}_{\ell}^{s} \cap E_{*}\left(\beta \times b_{\ell}^{s}\right)=\mathcal{E}(\beta) .
$$

Combining Theorem 2.10 with Proposition 2.9 yields the following cohomological formula for the Knill trace of a map $F: Y \times X \rightarrow X$ :

Proposition 2.11. For $y \in H_{n}(Y), L(F)(y)=(-1)^{n} L_{n}\left(\bar{\lambda}\left(\hat{F}_{*}(y)\right)\right)$.

\section{A Higher Hopf Trace Formula}

Let $\left(V_{*}, \partial\right)$ be a finite dimensional chain complex over a field $\mathbb{F}$. This means $V_{*}$ is a graded vector space over $\mathbb{F}, \sum_{i} \operatorname{dim} V^{i}<\infty$ and $\partial: V_{*} \rightarrow V_{*}$ is a degree -1 homomorphism such that $\partial^{2}=0$. The dual of $\left(V_{*}, \partial\right)$ is the cochain complex, $\left(V^{*}, d\right)$, defined as follows: $V^{i}=\operatorname{Hom}_{\mathbb{F}}\left(V_{i}, \mathbb{F}\right)$ and the degree 1 homomorphism $d: V^{*} \rightarrow V^{*}$ is the transpose of $\partial$, i.e. $\langle d x, y\rangle=\langle x, \partial y\rangle$ for $x \in V^{i}$ and $y \in V_{i+1}$. Let $\left(C_{*}, \partial^{\prime}\right)$ be another (not necessarily finite dimensional) chain complex. Suppose 
$V_{*}$ has a cap product taking values in $C_{*}$ (see $\S 1$ ). We say that this cap product is compatible if the differential $\partial^{\prime}$ has the following derivation property:

$$
\partial^{\prime}(x \cap y)=x \cap \partial y+(-1)^{|y|-|x|} d x \cap y
$$

where $|\cdot|$ denotes the degree of a homogeneous element of $V_{*}$ or $V^{*}$. It is straightforward to verify that a compatible cap product induces a cap product $H^{r}\left(V^{*}\right) \otimes$ $H_{s}\left(V_{*}\right) \rightarrow H_{s-r}\left(C_{*}\right)$.

Recall that a degree $n$ homomorphism $\theta: V_{*} \rightarrow V_{*}$ is a chain map if $\theta \partial=(-1)^{n} \partial \theta$ in which case $\theta$ induces a homomorphism of degree $n$ on homology which we denote by $H_{*}(\theta): H_{*}\left(V_{*}\right) \rightarrow H_{*}\left(V_{*}\right)$.

Theorem 3.1 (Higher Hopf Trace Formula). Let $\left(V_{*}, \partial\right)$ be a finite dimensional chain complex over $\mathbb{F}$ with a given compatible cap product taking values in $\left(C_{*}, \partial^{\prime}\right)$. Let $\theta: V_{*} \rightarrow V_{*}$ be a degree $n$ chain map. Then $L_{n}(\theta) \in C_{n}$ is a cycle representing the homology class $L_{n}\left(H_{*}(\theta)\right) \in H_{n}\left(C_{*}\right)$.

Proof. For each $k$ choose vectors $e_{1}^{k}, \ldots, e_{m_{k}}^{k} \in V_{k}$ and $f_{1}^{k}, \ldots, f_{n_{k}}^{k} \in V_{k}$ so that

1. $\left\{\partial e_{1}^{k+1}, \ldots, \partial e_{m_{k+1}}^{k+1}\right\}$ is a basis for $B_{k} \equiv \operatorname{Im}\left(\partial: V_{k+1} \rightarrow V_{k}\right)$,

2. $\left\{\partial e_{1}^{k+1}, \ldots, \partial e_{m_{k+1}+1}^{k+1}, f_{1}^{k}, \ldots, f_{n_{k}}^{k}\right\}$ is a basis for $Z_{k} \equiv \operatorname{ker}\left(\partial: V_{k} \rightarrow V_{k-1}\right)$,

3. $\left\{\partial e_{1}^{k+1}, \ldots, \partial e_{m_{k+1}}^{k+1}, f_{1}^{k}, \ldots, f_{n_{k}}^{k}, e_{1}^{k}, \ldots, e_{m_{k}}^{k}\right\}$ is a basis for $V_{k}$.

By Proposition 1.2, we have

$$
L_{n}(\theta)=\sum_{k}(-1)^{k+n}\left(\sum_{j=1}^{m_{k+1}} \overline{\partial e_{j}^{k+1}} \cap \theta\left(\partial e_{j}^{k+1}\right)+\sum_{j=1}^{n_{k}} \overline{f_{j}^{k}} \cap \theta\left(f_{j}^{k}\right)+\sum_{j=1}^{m_{k}} \overline{e_{j}^{k}} \cap \theta\left(e_{j}^{k}\right)\right) .
$$

Let $y=e_{j}^{k+1}$. Note that $d(\overline{\partial y})=y$. We have

$$
\begin{aligned}
\partial^{\prime}(\overline{\partial y} \cap \theta(y)) & =\overline{\partial y} \cap \partial \theta(y)+(-1)^{(k+1+n)-k} d(\overline{\partial y}) \cap \theta(y) \\
& =(-1)^{n} \overline{\partial y} \cap \theta(\partial y)+(-1)^{1+n} y \cap \theta(y) \\
& =(-1)^{n}(\overline{\partial y} \cap \theta(\partial y)-y \cap \theta(y)) .
\end{aligned}
$$

Using this identity in the above expression for $L_{n}(\theta)$, we obtain

$$
L_{n}(\theta)=\sum_{k}(-1)^{k+n} \sum_{j=1}^{n_{k}} \overline{f_{j}^{k}} \cap \theta\left(f_{j}^{k}\right)+\partial^{\prime} u
$$

where $u \in C_{n+1}$. Now $\partial^{\prime}\left(\bar{f}_{j}^{k} \cap \theta\left(f_{j}^{k}\right)\right)=\bar{f}_{j}^{k} \cap \partial \theta\left(f_{j}^{k}\right)+(-1)^{n} d \bar{f}_{j}^{k} \cap \theta\left(f_{j}^{k}\right)$. Since $\partial f_{j}^{k}=0, \partial \theta\left(f_{j}^{k}\right)=(-1)^{n} \theta\left(\partial f_{j}^{k}\right)$ and $d \bar{f}_{j}^{k}=0$, we conclude $\partial\left(\bar{f}_{j}^{k} \cap \theta\left(f_{j}^{k}\right)\right)=0$ and so $L_{n}(\theta)$ is a cycle. Observe that for each $k,\left\{f_{1}^{k}, \ldots, f_{n_{k}}^{k}\right\}$ projects to a basis for $H_{k}\left(V_{*}\right) \equiv Z_{k} / B_{k}$. Using this basis for $H_{*}\left(V_{*}\right)$ and applying Proposition 1.2 to the computation $L_{n}\left(H_{*}(\theta)\right)$, the conclusion of the theorem easily follows.

Remark. Suppose $n=0$ and that the cap product is as in Remark 1.4. If $\mathbb{F}$ has characteristic 0 and $c_{0}$ is a cycle projecting to a non-zero homology class in $H_{0}\left(C_{*}\right)$, then Theorem 3.1 recovers the classical Hopf trace formula.

The following "dual" version of Theorem 3.1 is proved similarly: 
Theorem 3.2. Let $\left(V_{*}, \partial\right)$ be a finite dimensional chain complex over $\mathbb{F}$ with a given compatible cap product taking values in $\left(C_{*}, \partial^{\prime}\right)$. Let $\theta: V^{*} \rightarrow V^{*}$ be a degree $-n$ cochain map. Then $L_{n}(\theta) \in C_{n}$ is a cycle representing the homology class $L_{n}\left(H^{*}(\theta)\right) \in H_{n}\left(C_{*}\right)$.

The Lefschetz number of a cellular map $f: X \rightarrow X$ of a finite CW complex can be calculated using cellular chains: $L(f)=\sum_{i \geq 0}(-1)^{i} \operatorname{trace}\left(f_{i}: C_{i}(X) \rightarrow C_{i}(X)\right)$ where $C_{i}(X)$ is the free abelian group of cellular $i$-chains and $f_{i}: C_{i}(X) \rightarrow C_{i}(X)$ is the homomorphism induced by $f$. The Knill trace can also be computed using chains although one must employ simplicial chains because of the need for a chain level cap product.

Let $K$ be an ordered simplicial complex (i.e. there is given a partial order of the vertex set, $K_{0}$, such that each simplex is totally ordered). Serving as a ring of coefficients, let $R$ be a principal ideal domain. Define the chain complex $\left(C_{*}^{\prime}(K ; R), \partial^{\prime}\right)$ as follows: $C_{n}^{\prime}(K, R)$ is the free $R$-module generated by $n+1$-tuples $\left(v_{0}, \ldots, v_{n}\right) \in K_{0}^{n+1}$ such that $\left\{v_{0}, \ldots, v_{n}\right\}$ spans a simplex of $K$ and $v_{0} \leq v_{1} \leq$ $\ldots \leq v_{n}$ in the given ordering. The boundary operator is defined in the usual way by $\partial^{\prime}\left(v_{0}, \ldots, v_{n}\right)=\sum_{i=0}^{n}(-1)^{i}\left(v_{0}, \ldots, \hat{v}_{i}, \ldots, v_{n}\right)$ where " $\hat{v}_{i}$ " indicates that $v_{i}$ is omitted. For nonempty $K$, this chain complex is always of infinite rank over $R$. Let $D_{*} \subset C_{*}^{\prime}(K ; R)$ be generated by the degenerate simplices, i.e. $n^{-}$ tuples of the form $\left(v_{0}, \ldots, v_{n}\right) \in C_{n}^{\prime}(K ; R)$ where $v_{i}=v_{i+1}$ for some $i$. It is easy to check that $D_{*}$ is a subcomplex. Define $C_{*}(K ; R)$ to be the quotient complex $C_{*}^{\prime}(K ; R) / D_{*}$. Then $C_{n}(K ; R)$ is a free $R$-module of rank equal to the number of (nondegenerate) $n$-simplices of $K$. Given two ordered simplicial complexes $L$ and $K$, their product $L \times K$ is the ordered simplicial complex defined as follows. The vertex set of $L \times K$ is $L_{0} \times K_{0}$ equipped with the left-to-right lexicographic ordering (i.e. $(a, b) \leq(c, d)$ if and only if $a<c$ or $a=c$ and $b \leq d$ ). Distinct vertices $\left(v_{0}, w_{0}\right), \ldots,\left(v_{n}, w_{n}\right) \in L_{0} \times K_{0}$ with $\left(v_{0}, w_{0}\right)<\left(v_{1}, w_{1}\right)<\cdots<\left(v_{n}, w_{n}\right)$ span an $n$-simplex of $L \times K$ if

1. $\left\{v_{0}, \ldots, v_{n}\right\}$ spans a simplex of $L$ and $v_{0} \leq v_{1} \cdots \leq v_{n}$,

2. $\left\{w_{0}, \ldots, w_{n}\right\}$ spans a simplex of $K$ and $w_{0} \leq w_{1} \cdots \leq w_{n}$.

The shuffle product (see [M, p. 243]) provides a natural chain equivalence

$$
C_{*}(L ; R) \otimes C_{*}(K ; R) \stackrel{\mathcal{S}}{\longrightarrow} C_{*}(L \times K ; R)
$$

from which we obtain a natural chain map

$$
C_{*}(L ; R) \stackrel{\hat{\mathcal{S}}}{\longrightarrow} \operatorname{Hom}\left(C_{*}(K ; R), C_{*}(L \times K ; R)\right) .
$$

Now suppose that $K$ is finite and $F:|L| \times|K| \rightarrow|K|$ is a continuous map where "| |" denotes geometric realization. There is a natural homeomorphism $|L| \times|K| \cong \mid L \times$ $K \mid$. Let $F^{\prime}:(L \times K)^{\prime} \rightarrow K$ be a simplicial approximation to $F$ where $(L \times K)^{\prime}$ is a subdivision of $L \times K$. Let $F_{*}^{\prime \prime}: C_{*}(L \times K ; R) \rightarrow C_{*}(K ; R)$ be the composite of a subdivision chain map sd: $C_{*}(L \times K ; R) \rightarrow C_{*}\left((L \times K)^{\prime} ; R\right)$ and the induced chain map $F_{*}^{\prime}: C_{*}\left((L \times K)^{\prime} ; R\right) \rightarrow C_{*}(K ; R)$. Let $\mu: C_{*}(L ; R) \rightarrow \operatorname{Hom}\left(C_{*}(K ; R), C_{*}(K ; R)\right)$ be the composite of $\hat{\mathcal{S}}: C_{*}(L ; R) \rightarrow \operatorname{Hom}\left(C_{*}(K ; R), C_{*}(L \times K ; R)\right)$ and the chain map

$$
\operatorname{Hom}\left(C_{*}(K ; R), C_{*}(L \times K ; R)\right) \rightarrow \operatorname{Hom}\left(C_{*}(K ; R), C_{*}(K ; R)\right)
$$


induced by $F_{*}^{\prime \prime}$. Define $C^{*}(K ; R)=\operatorname{Hom}_{R}\left(C_{*}(K ; R), R\right)$. The cap product

$$
C^{k}(K ; R) \otimes C_{n+k}(K ; R) \stackrel{\cap}{\longrightarrow} C_{n}(K ; R)
$$

is given explicitly by

$$
f \cap\left[v_{0}, \ldots, v_{n+k}\right]=f\left(\left[v_{n}, \ldots, v_{k+n}\right]\right)\left[v_{0}, \ldots, v_{n}\right]
$$

where we use the notation $\left[w_{0}, \ldots, w_{m}\right]$ for the image in $C_{m}(K ; R)$ of $\left(w_{0}, \ldots, w_{m}\right)$ $\in C_{m}^{\prime}(K ; R)$. The theory of $\S 1$ is clearly valid for free modules over $R$ (in place of vector spaces over a field $\mathbb{F}$ ) and so we make the following definition.

Definition 3.3. The chain level Knill trace of $F^{\prime}:(L \times K)^{\prime} \rightarrow K$ is the degree 0 homomorphism $L\left(F^{\prime}\right): C_{*}(L ; R) \rightarrow C_{*}(K ; R)$ given by $L\left(F^{\prime}\right)(z)=L_{n}(\mu(z))$ for $z \in C_{n}(L ; R)$.

It is straightforward to verify that $L\left(F^{\prime}\right)$ is a chain map. If $R=\mathbb{F}$ is a field, the Higher Hopf Trace Formula (Theorem 3.1) yields:

Proposition 3.4. The chain level Knill trace $L\left(F^{\prime}\right): C_{*}(L ; \mathbb{F}) \rightarrow C_{*}(K ; \mathbb{F})$ induces the Knill trace $L(F): H_{*}(|L|) \rightarrow H_{*}(|K|)$.

Remark. One can think of $F_{*}^{\prime \prime}$ as being induced by a cellular map $F^{\prime \prime}:|L| \times|K| \rightarrow$ $|K|$ and of the chain level Knill trace as " $L\left(F^{\prime \prime}\right)$ ".

\section{Sum and Product Theorems}

Consider a topological pair $(X, A)$ such that $H_{*}(A)$ and $H_{*}(X)$ are both finite dimensional over $\mathbb{F}$. Using the homology long exact sequence for the pair $(X, A)$, we see that $H_{*}(X, A)$ is necessarily finite dimensional. Let $F: Y \times(X, A) \rightarrow(X, A)$ be a map of pairs. Write $F_{X}$ for $F$ regarded as a map $Y \times(X, \emptyset) \rightarrow(X, \emptyset), F_{X, A}$ for $F$ regarded as a map $Y \times(X, A) \rightarrow(X, A)$, and $F_{A}$ for the restriction of $F$ to $Y \times A$ regarded as a map $Y \times(A, \emptyset) \rightarrow(A, \emptyset)$. Let $i: A \hookrightarrow X$ be the inclusion. We have the following additivity property of the Knill trace.

Theorem 4.1 (Sum Theorem). $L\left(F_{X}\right)=i_{*}\left(L\left(F_{A}\right)\right)+L\left(F_{X, A}\right)$.

Proof. Let $j:(X, \emptyset) \hookrightarrow(X, A)$ be the inclusion. Let $\partial: H_{*}(X, A) \rightarrow H_{*}(A)$ denote the degree -1 boundary operator. Using the homology long exact sequence for the

pair $(X, A)$, for $k \geq 0$ choose linearly independent sets of vectors: $d_{1}^{k}, \ldots, d_{m_{k}}^{k} \in$ $H_{k}(A), f_{1}^{k}, \ldots, f_{n_{k}}^{k} \in H_{k}(X)$ and $v_{1}^{k}, \ldots, v_{p_{k}}^{k} \in H_{k}(X, A)$ so that for each $k \geq 0$ : $\partial v_{1}^{k+1}, \ldots, \partial v_{p_{k+1}}^{k+1}, d_{1}^{k}, \ldots, d_{m_{k}}^{k}$ is a basis for $H_{k}(A)$, $i_{*}\left(d_{1}^{k}\right), \ldots, i_{*}\left(d_{m_{k}}^{k}\right), f_{1}^{k}, \ldots, f_{n_{k}}^{k}$ is a basis for $H_{k}(X)$ and $j_{*}\left(f_{1}^{k}\right), \ldots, j_{*}\left(f_{n_{k}}^{k}\right), v_{1}^{k}, \ldots, v_{p_{k}}^{k}$ is a basis for $H_{k}(X, A)$.

Notation. Given a basis $w_{1}, \ldots, w_{m}$ for a homology group, let $\bar{w}_{1}, \ldots, \bar{w}_{m}$ denote the dual basis for the corresponding cohomology group, i.e. $\left\langle\bar{w}_{k}, w_{\ell}\right\rangle=\delta_{k, \ell}$ (Kronecker delta).

Let $u \in H_{n}(Y)$. Then by Proposition 1.2:

$$
\begin{aligned}
L\left(F_{X}\right)(u)=\sum_{k \geq 0}(-1)^{k+n}\left(\sum_{\ell=1}^{m_{k}} \overline{i_{*}\left(d_{\ell}^{k}\right)} \cap\left(F_{X}\right)_{*}\left(u \times i_{*}\left(d_{\ell}^{k}\right)\right)\right. & \\
& \left.+\sum_{\ell=1}^{n_{k}} \bar{f}_{\ell}^{k} \cap\left(F_{X}\right)_{*}\left(u \times f_{\ell}^{k}\right)\right),
\end{aligned}
$$




$$
\begin{gathered}
L\left(F_{A}\right)(u)=\sum_{k \geq 0}(-1)^{k+n}\left(\sum_{\ell=1}^{p_{k+1}} \overline{\partial v_{\ell}^{k+1}} \cap\left(F_{A}\right)_{*}\left(u \times \partial v_{\ell}^{k+1}\right)\right. \\
\left.+\sum_{\ell=1}^{m_{k}} \bar{d}_{\ell}^{k} \cap\left(F_{A}\right)_{*}\left(u \times d_{\ell}^{k}\right)\right), \\
L\left(F_{X, A}\right)(u)=\sum_{k \geq 0}(-1)^{k+n}\left(\sum_{\ell=1}^{n_{k}} \overline{j_{*}\left(f_{\ell}^{k}\right)} \cap\left(F_{X, A}\right)_{*}\left(u \times j_{*}\left(f_{\ell}^{k}\right)\right)\right. \\
\left.+\sum_{\ell=1}^{p_{k}} \bar{v}_{\ell}^{k} \cap\left(F_{X, A}\right)_{*}\left(u \times v_{\ell}^{k}\right)\right) .
\end{gathered}
$$

Note that $j F_{X}=F_{X, A} \circ(\mathrm{id} \times j)$. We have:

$$
\begin{aligned}
\overline{j_{*}\left(f_{\ell}^{k}\right)} \cap\left(F_{X, A}\right)_{*}\left(u \times j_{*}\left(f_{\ell}^{k}\right)\right) & =\overline{j_{*}\left(f_{\ell}^{k}\right)} \cap j_{*}\left(F_{X}\right)_{*}\left(u \times f_{\ell}^{k}\right) \\
& =j^{*}\left(\overline{j_{*}\left(f_{\ell}^{k}\right)}\right) \cap\left(F_{X}\right)_{*}\left(u \times f_{\ell}^{k}\right) \\
& =\bar{f}_{\ell}^{k} \cap\left(F_{X}\right)_{*}\left(u \times f_{\ell}^{k}\right) .
\end{aligned}
$$

Note that $i F_{A}=F_{X} \circ($ id $\times i)$. We have:

$$
\begin{aligned}
\overline{i_{*}\left(d_{\ell}^{k}\right)} \cap\left(F_{X}\right)_{*}\left(u \times i_{*}\left(d_{\ell}^{k}\right)\right) & =\overline{i_{*}\left(d_{\ell}^{k}\right)} \cap i_{*}\left(F_{A}\right)_{*}\left(u \times d_{\ell}^{k}\right) \\
& =i_{*}\left(i^{*}\left(\overline{i_{*}\left(d_{\ell}^{k}\right)}\right) \cap\left(F_{A}\right)_{*}\left(u \times d_{\ell}^{k}\right)\right) \\
& =i_{*}\left(\bar{d}_{\ell}^{k} \cap\left(F_{A}\right)_{*}\left(u \times d_{\ell}^{k}\right)\right) .
\end{aligned}
$$

The naturality of the homology long exact sequence of a pair implies $\left(F_{A}\right)_{*}(u \times \partial x)=(-1)^{n} \partial\left(F_{X, A}\right)_{*}(u \times x)$ for all $x \in H_{*}(X, A)$. Let $d: H^{*}(A) \rightarrow$ $H^{*}(X, A)$ denote the degree 1 coboundary operator; it has the properties: $\langle d z, w\rangle=$ $\langle z, \partial w\rangle$ for all $z \in H^{k}(X)$ and $w \in H_{k+1}(X, A)$, and $d\left(i^{*}(z) \cup \beta\right)=(-1)^{k} z \cup d \beta$ for all $z \in H^{k}(X)$ and $\beta \in H^{*}(A)$ (see [Sp, 5.6.12]). For all $z \in H^{n}(X)$ we have:

$$
\begin{aligned}
\left\langle z, i_{*}\right. & \left.\left(\overline{\partial v_{\ell}^{k+1}} \cap\left(F_{A}\right)_{*}\left(u \times \partial v_{\ell}^{k+1}\right)\right)\right\rangle=(-1)^{n}\left\langle z, i_{*}\left(\overline{\partial v_{\ell}^{k+1}} \cap \partial\left(F_{X, A}\right)_{*}\left(u \times v_{\ell}^{k+1}\right)\right)\right\rangle \\
& =(-1)^{n}\left\langle i^{*}(z) \cup \overline{\partial v_{\ell}^{k+1}}, \partial\left(F_{X, A}\right)_{*}\left(u \times v_{\ell}^{k+1}\right)\right\rangle \\
& =(-1)^{n}\left\langle d\left(i^{*}(z) \cup \overline{\partial v_{\ell}^{k+1}}\right),\left(F_{X, A}\right)_{*}\left(u \times v_{\ell}^{k+1}\right)\right\rangle \\
& =(-1)^{n}(-1)^{n}\left\langle z \cup d\left(\overline{\partial v_{\ell}^{k+1}}\right),\left(F_{X, A}\right)_{*}\left(u \times v_{\ell}^{k+1}\right)\right\rangle \\
& =\left\langle z \cup \bar{v}_{\ell}^{k+1},\left(F_{X, A}\right)_{*}\left(u \times v_{\ell}^{k+1}\right)\right\rangle \\
& =\left\langle z, \bar{v}_{\ell}^{k+1} \cap\left(F_{X, A}\right)_{*}\left(u \times v_{\ell}^{k+1}\right)\right\rangle .
\end{aligned}
$$

It follows that $i_{*}\left(\overline{\partial v_{\ell}^{k+1}} \cap\left(F_{A}\right)_{*}\left(u \times \partial v_{\ell}^{k+1}\right)\right)=\bar{v}_{\ell}^{k+1} \cap\left(F_{X, A}\right)_{*}\left(u \times v_{\ell}^{k+1}\right)$. 
Combining the above identities, we have:

$$
\begin{aligned}
& i_{*}\left(L\left(F_{A}\right)\right)(u)+L\left(F_{X, A}\right)(u) \\
& \left.=\sum_{k \geq 0}(-1)^{k+n}\left(\sum_{\ell=1}^{p_{k+1}} i_{*} \overline{\partial v_{\ell}^{k+1}} \cap\left(F_{A}\right)_{*}\left(u \times \partial v_{\ell}^{k+1}\right)\right)+\sum_{\ell=1}^{m_{k}} i_{*}\left(\bar{d}_{\ell}^{k} \cap\left(F_{A}\right)_{*}\left(u \times d_{\ell}^{k}\right)\right)\right) \\
& \quad+\sum_{k \geq 0}(-1)^{k+n}\left(\sum_{\ell=1}^{n_{k}} \overline{j_{*}\left(f_{\ell}^{k}\right)} \cap\left(F_{X, A}\right)_{*}\left(u \times j_{*}\left(f_{\ell}^{k}\right)\right)+\sum_{\ell=1}^{p_{k}} \bar{v}_{\ell}^{k} \cap\left(F_{X, A}\right)_{*}\left(u \times v_{\ell}^{k}\right)\right) \\
& =\sum_{k \geq 0}(-1)^{k+n}\left(\sum_{\ell=1}^{p_{k+1}} \bar{v}_{\ell}^{k+1} \cap\left(F_{X, A}\right)_{*}\left(u \times v_{\ell}^{k+1}\right)+\sum_{\ell=1}^{m_{k}} \overline{i_{*}\left(d_{\ell}^{k}\right)} \cap\left(F_{X}\right)_{*}\left(u \times i_{*}\left(d_{\ell}^{k}\right)\right)\right) \\
& \quad+\sum_{k \geq 0}(-1)^{k+n}\left(\sum_{\ell=1}^{n_{k}} \bar{f}_{\ell}^{k} \cap\left(F_{X}\right)_{*}\left(u \times f_{\ell}^{k}\right)+\sum_{\ell=1}^{p_{k}} \bar{v}_{\ell}^{k} \cap\left(F_{X, A}\right)_{*}\left(u \times v_{\ell}^{k}\right)\right) \\
& =\sum_{k \geq 0}(-1)^{k+n}\left(\sum_{\ell=1}^{m_{k}} \overline{i_{*}\left(d_{\ell}^{k}\right)} \cap\left(F_{X}\right)_{*}\left(u \times i_{*}\left(d_{\ell}^{k}\right)\right)+\sum_{\ell=1}^{n_{k}} \bar{f}_{\ell}^{k} \cap\left(F_{X}\right)_{*}\left(u \times f_{\ell}^{k}\right)\right) \\
& =L\left(F_{X}\right)(u) . \quad \square
\end{aligned}
$$

Let $X$ be a space with a finite filtration $\emptyset=X_{-1} \subset X_{0} \subset \cdots \subset X_{m}=X$ such that for each $0 \leq j \leq m, H_{*}\left(X_{j}, X_{j-1}\right)$ is finite dimensional. Suppose $F: Y \times X \rightarrow$ $X$ is filtration preserving, i.e. $F\left(Y \times X_{j}\right) \subset X_{j}$. Let $F_{j, j-1}$ denote the restriction of $F$ to $Y \times X_{j}$ regarded as a map of pairs $Y \times\left(X_{j}, X_{j-1}\right) \rightarrow\left(X_{j}, X_{j-1}\right)$ and let $i_{j}: X_{j} \hookrightarrow X$ be the inclusion. Then repeated application of the Sum Theorem (Theorem 4.1) yields:

Corollary 4.2. $L(F)=\sum_{j=0}^{m}\left(i_{j}\right)_{*}\left(L\left(F_{j, j-1}\right)\right)$.

Let $F: Y \times X \rightarrow X$ and $p: X \rightarrow B$ be maps. We say $F$ is a fiber map if $p \circ F=p \circ p^{\prime}$ where $p^{\prime}: Y \times X \rightarrow X$ is projection. We also say $B$ is homology equivalent over $\mathbb{F}$ to a finite complex if there is a map $B^{\prime} \rightarrow B$, where $B^{\prime}$ is a finite $\mathrm{CW}$ complex, inducing an isomorphism on homology with $\mathbb{F}$ coefficients. As an application of Corollary 4.2, we prove the following product theorem for $L(F)$.

Theorem 4.3 (Product Theorem). Let $K \stackrel{i}{\rightarrow} X \stackrel{p}{\rightarrow} B$ be an orientable Serre fibration where $B$ is homology equivalent over $\mathbb{F}$ to a finite complex and $H_{*}(K)$ is finite dimensional. Let $F: Y \times X \rightarrow X$ be a fiber map. Then

$$
L(F)=\chi(B) i_{*}\left(L\left(\left.F\right|_{Y \times K}\right)\right)
$$

where $\chi(B) \equiv \sum_{i \geq 0}(-1)^{i} \operatorname{dim} H_{i}(B)$ is the Euler characteristic of $B$.

Note that the hypotheses of the theorem imply that $H_{*}(X)$ is finite dimensional.

Proof. By pulling back the fibration $p$ via a map $B^{\prime} \rightarrow B$, where $B^{\prime}$ is a finite CW complex, inducing an isomorphism on homology with $\mathbb{F}$ coefficients, we can assume without loss of generality that $B$ is a finite $\mathrm{CW}$ complex. Define a finite filtration of $X$ by $X_{k}=p^{-1}\left(B_{k}\right)$ where $B_{k}$ is the $k$-skeleton of $B$. Since $F$ is a fiber map, it preserves the filtration $\left\{X_{k}\right\}$. By Corollary $4.2, L(F)=\sum_{j \geq 0}\left(i_{j}\right)_{*}\left(L\left(F_{j, j-1}\right)\right)$. 
From the construction of the Serre spectral sequence (e.g. [W]), there is an isomorphism $H^{k}\left(X_{j}, X_{j-1}\right) \cong H^{k-j}(K) \otimes C^{j}(B)$, where $C^{j}(B)$ denotes the vector space with basis the $j$-cells of $B$. Moreover, because $F$ is a fiber map over $1_{B}$ : $B \rightarrow B, F_{j, j-1_{*}}=F_{0 *} \otimes 1_{C^{j}(B)}$, where $F_{0}=\left.F\right|_{Y \times K}$. For $j$ fixed, let $\left\{\bar{\gamma}_{i}^{r} \times \bar{b}_{l}^{j}\right\}$ be a basis for $H^{r+j}\left(X_{j}, X_{j-1}\right)$, where the $\gamma^{\prime}$ 's and $b$ 's are bases for $H^{r}(K)$ and $C^{j}(B)$ respectively. For $y \in H_{n}(Y)$ we have

$$
\begin{aligned}
\bar{\gamma}_{i}^{k-j} & \times \bar{b}_{l}^{j} \cap F_{j, j-1_{*}}\left(y \times\left(\gamma_{i}^{k-j} \times b_{l}^{j}\right)\right)=\bar{\gamma}_{i}^{k-j} \times \bar{b}_{l}^{j} \cap F_{0 *}\left(y \times \gamma_{i}^{k-j}\right) \times b_{l}^{j} \\
& =(-1)^{(k-j)(j-j)} \gamma_{i}^{k-j} \cap F_{0 *}\left(y \times \gamma_{i}^{k-j}\right) \times \bar{b}_{l}^{j} \cap b_{l}^{j} \\
& =\gamma_{i}^{k-j} \cap F_{0 *}\left(y \times \gamma_{i}^{k-j}\right) \times 1 .
\end{aligned}
$$

Since this is true for each $\bar{b}_{l}^{j}$, we obtain

$$
\begin{aligned}
& \sum_{j_{k}\left(X_{j}, X_{j-1}\right)} \bar{\gamma}_{i}^{k-j} \times \bar{b}_{l}^{j} \cap F_{j, j-1_{*}}\left(y \times\left(\gamma_{i}^{k-j} \times b_{l}^{j}\right)\right) \\
& \quad=\sum_{j_{k}\left(X_{j}, X_{j-1}\right)} \bar{\gamma}_{i}^{k-j} \cap F_{0 *}\left(y \times \gamma_{i}^{k-j}\right) \cdot \operatorname{dim} C^{j}(B)
\end{aligned}
$$

and

$$
\begin{aligned}
L\left(F_{j, j-1}\right)(y) & =\sum_{k \geq 0}(-1)^{k+n} \sum_{j_{k}\left(X_{j}, X_{j-1}\right)} \bar{\gamma}_{i}^{k-j} \times \bar{b}_{l}^{j} \cap F_{j, j-1_{*}}\left(y \times\left(\gamma_{i}^{k-j} \times b_{l}^{j}\right)\right. \\
& =\sum_{k \geq 0}(-1)^{k+n} \sum_{j_{k-j}(K)} \bar{\gamma}_{i}^{k-j} \cap F_{0 *}\left(y \times \gamma_{i}^{k-j}\right) \cdot \operatorname{dim} C^{j}(B) .
\end{aligned}
$$

Finally, using $L(F)=\sum_{j \geq 0}\left(i_{j}\right)_{*}\left(L\left(F_{j, j-1}\right)\right)$ and noting that the isomorphism above identifies $\left(i_{j}\right)_{*}\left(L\left(F_{j, j-1}\right)\right)$ with $i_{*}\left(\sum_{k \geq 0}(-1)^{k+n} \sum_{j_{k-j}(K)} \bar{\gamma}_{i}^{k-j} \cap F_{0 *}\left(y \times \gamma_{i}^{k-j}\right)\right.$. $\operatorname{dim} C^{j}(B)$, we have

$$
\begin{aligned}
L(F) & =\sum_{j \geq 0} \sum_{k \geq 0}(-1)^{k+n} \sum_{j_{k-j}(K)} i_{*}\left(\bar{\gamma}_{i}^{k-j} \cap F_{0 *}\left(y \times \gamma_{i}^{k-j}\right)\right) \cdot \operatorname{dim} C^{j}(B) \\
& =\sum_{j \geq 0}(-1)^{j} \operatorname{dim} C^{j}(B) \sum_{k \geq 0}(-1)^{k-j+n} \sum_{j_{k-j}(K)} i_{*}\left(\bar{\gamma}_{i}^{k-j} \cap F_{0 *}\left(y \times \gamma_{i}^{k-j}\right)\right) \\
& =\sum_{j \geq 0}(-1)^{j} \operatorname{dim} C^{j}(B) \sum_{k-j \geq 0}(-1)^{k-j+n} \sum_{j_{k-j}(K)} i_{*}\left(\bar{\gamma}_{i}^{k-j} \cap F_{0 *}\left(y \times \gamma_{i}^{k-j}\right)\right)
\end{aligned}
$$

since nonzero terms occur only for $k-j \geq 0$;

$$
\begin{aligned}
& =\chi(B) \cdot i_{*} L\left(F_{0}\right) \\
& =\chi(B) \cdot i_{*} L\left(\left.F\right|_{Y \times K}\right) .
\end{aligned}
$$

Remark 4.4. Suppose that, in the above theorem, $F: Y \times X \rightarrow X$ lies over $f$ : $B \rightarrow B$. Then the proof given above goes through with a slight change in the first equality

$$
\bar{\gamma}_{i}^{k-j} \times \bar{b}_{l}^{j} \cap F_{j, j-1_{*}}\left(y \times\left(\gamma_{i}^{k-j} \times b_{l}^{j}\right)\right)=\bar{\gamma}_{i}^{k-j} \times \bar{b}_{l}^{j} \cap F_{0 *}\left(y \times \gamma_{i}^{k-j}\right) \times f_{*}\left(b_{l}^{j}\right) .
$$

The only effect this has on the calculations is to introduce terms such as $\bar{b}_{l}^{j} \cap f_{*}\left(b_{l}^{j}\right)$ in place of 1 . But it is easy to see that these terms produce the Lefschetz number of $B$ instead of the Euler characteristic. Hence, for $F: Y \times X \rightarrow X$ lying over $f: B \rightarrow B$, we have the formula $L(F)=L(f) \cdot i_{*} L\left(\left.F\right|_{Y \times K}\right)$.

We also have the following general product theorem: 
Theorem 4.5. Let $Y_{1}$ and $Y_{2}$ be parameter spaces. Let $X_{1}$ and $X_{2}$ be spaces with finite dimensional homology over $\mathbb{F}$. For any maps $F_{1}: Y_{1} \times X_{1} \rightarrow X_{1}, F_{2}: Y_{2} \times$ $X_{2} \rightarrow X_{2}$ there is a commutative diagram:

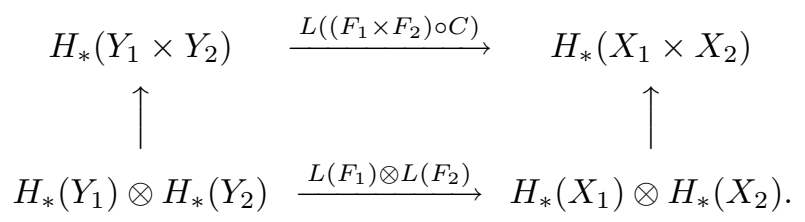

where $C: Y_{1} \times Y_{2} \times X_{1} \times X_{2} \rightarrow Y_{1} \times X_{1} \times Y_{2} \times X_{2}$ is the map which interchanges the middle factors and the vertical arrows are given by the Künneth isomorphism.

Proof. We must show $L\left(\left(F_{1} \times F_{2}\right) \circ C\right)(u \times v)=L\left(F_{1}\right)(u) \times L\left(F_{2}\right)(v)$ where $u \in$ $H_{p}\left(Y_{1}\right)$ and $v \in H_{q}\left(Y_{2}\right)$. For each $k \geq 0$, let $b_{1}^{k}, \ldots, b_{m_{k}}^{k}$ be a basis for $H_{k}\left(X_{1}\right)$ and let $c_{1}^{k}, \ldots, c_{n_{k}}^{k}$ be a basis for $H_{k}\left(X_{1}\right)$. The corresponding dual bases for cohomology will be denoted by $\bar{b}_{1}^{k}, \ldots, \bar{b}_{m_{k}}^{k}$ and $\bar{c}_{1}^{k}, \ldots, \bar{c}_{n_{k}}^{k}$ respectively. Then $\left\{b_{i}^{s} \times c_{j}^{k-s} \mid 0 \leq\right.$ $\left.s \leq k, 1 \leq i \leq m_{s}, 1 \leq j \leq n_{k-s}\right\}$ is a basis for $H_{k}\left(X_{1} \times X_{2}\right)$; the corresponding dual basis for $H^{k}\left(X_{1} \times X_{2}\right)$ is $\left\{\bar{b}_{i}^{s} \times \bar{c}_{j}^{k-s}\right\}$. We have:

$$
\begin{aligned}
L\left(\left(F_{1} \times\right.\right. & \left.\left.F_{2}\right) \circ C\right)(u \times v) \\
= & \sum_{k \geq 0}(-1)^{k+p+q} \sum_{s, i, j}\left(\bar{b}_{i}^{s} \times \bar{c}_{j}^{k-s}\right) \cap\left(\left(F_{1} \times F_{2}\right) \circ C\right)_{*}\left((u \times v) \times\left(b_{i}^{s} \times c_{j}^{k-s}\right)\right) \\
= & \sum_{k \geq 0}(-1)^{k+p+q} \sum_{s, i, j}(-1)^{q s}\left(\bar{b}_{i}^{s} \times \bar{c}_{j}^{k-s}\right) \cap\left(\left(F_{1}\right)_{*}\left(u \times b_{i}^{s}\right) \times\left(F_{2}\right)_{*}\left(v \times c_{j}^{k-s}\right)\right) \\
= & \sum_{k \geq 0}(-1)^{k+p+q} \sum_{s, i, j}(-1)^{q s}(-1)^{s q}\left(\bar{b}_{i}^{s} \cap\left(F_{1}\right)_{*}\left(u \times b_{i}^{s}\right)\right) \\
& \times\left(\bar{c}_{j}^{k-s} \cap\left(F_{2}\right)_{*}\left(v \times c_{j}^{k-s}\right)\right) \\
= & \left(\sum_{k \geq 0}(-1)^{k+p} \sum_{i} \bar{b}_{i}^{k} \cap\left(F_{1}\right)_{*}\left(u \times b_{i}^{k}\right)\right) \\
& \times\left(\sum_{k \geq 0}(-1)^{k+q} \sum_{j} \bar{c}_{j}^{k} \cap\left(F_{2}\right)_{*}\left(v \times c_{j}^{k}\right)\right) \\
= & L\left(F_{1}\right)(u) \times L\left(F_{2}\right)(v) .
\end{aligned}
$$

\section{Spherical Lefschetz and Euler Characteristics}

For a space $X$ and a self-map $f: X \rightarrow X$, consider the homotopy groups $\pi_{n}\left(X^{X}, f\right), n>0$, and the pointed set $\pi_{0}\left(X^{X}, f\right)$. The evaluation map ev: $X^{X} \rightarrow$ $X, g \mapsto g\left(x_{0}\right)$, induces $\mathrm{ev}_{n}: \pi_{n}\left(X^{X}, f\right) \rightarrow \pi_{n}\left(X, f\left(x_{0}\right)\right)$. We define, for $n \geq 1$, the higher Jiang group $J_{n}(X, f) \subset \pi_{n}\left(X, f\left(x_{0}\right)\right)$ to be the image of $\mathrm{ev}_{n}: \pi_{n}\left(X^{X}, f\right) \rightarrow$ $\pi_{n}\left(X, f\left(x_{0}\right)\right)$. When $f=$ id we write $G_{n}(X)$ for $J_{n}(X, f)$ and note that this is the $n^{\text {th }}$ Gottlieb group of $X$.

For $n>0$, choose the generator of $H_{n}\left(S^{n} ; \mathbb{Z}\right) \cong \mathbb{Z}$ corresponding to the "standard" orientation of $S^{n}$; more precisely, an ordered basis $\left\{b_{1}, \ldots, b_{n}\right\}$ for the tangent 
space of $S^{n} \subset \mathbb{R}^{n+1}$ at the point $v \in S^{n}$ is positively oriented if $\left\{e, b_{1}, \ldots, b_{n}\right\}$ is a positively oriented basis for the tangent space at $v$ of $\mathbb{R}^{n+1}$ where $e$ is an outward unit normal vector and $\mathbb{R}^{n+1}$ has the orientation determined by the standard basis. Let $U \in H_{n}\left(S^{n}\right)$ be the image of this standard generator under the coefficient homomorphism $H_{n}\left(S^{n} ; \mathbb{Z}\right) \rightarrow H_{n}\left(S^{n}\right)$. In case $n=0, S^{0}=\{-1,1\} \subset \mathbb{R}$ and we choose $1 \in S^{0}$ as the basepoint and define $U \in H_{0}\left(S^{0}\right) \cong \mathbb{F} \oplus \mathbb{F}$ to be the homology class represented by $-1 \in S^{0}$. The class $U \in H_{n}\left(S^{n}\right)$, denoted by $\left[S^{n}\right]$ in the introduction, is called the fundamental homology class of $S^{n}$.

Definition 5.1. Suppose $H_{*}(X)$ is finite dimensional. The $n^{\text {th }}$ spherical Lefschetz characteristic of $X$ based at $f: X \rightarrow X$ is the function $\Lambda_{n}(f ; \mathbb{F}): \pi_{n}\left(X^{X}, f\right) \rightarrow$ $H_{n}(X)$ given by $\Lambda_{n}(f ; \mathbb{F})([\hat{\gamma}])=L(\gamma)(U)$ where $\hat{\gamma}: S^{n} \rightarrow X^{X}$ determines $\gamma: S^{n} \times$ $X \rightarrow X$ via $\hat{\gamma}(s)(x)=\gamma(s, x)$. In case $f=\mathrm{id}_{X}$, the identity map of $X$, we write $\chi_{n}(X ; \mathbb{F}) \equiv \Lambda_{n}\left(\operatorname{id}_{X} ; \mathbb{F}\right)$ and call $\chi_{n}(X ; \mathbb{F})$ the $n^{\text {th }}$ spherical Euler characteristic of $X$.

By Proposition $1.2, \Lambda_{n}(f ; \mathbb{F})([\hat{\gamma}])$ is given by the formula:

$$
\Lambda_{n}(f ; \mathbb{F})([\hat{\gamma}])=\sum_{k \geq 0}(-1)^{k+n} \sum_{j=1}^{\beta_{k}} \bar{b}_{j}^{k} \cap \gamma_{*}\left(U \times b_{j}^{k}\right)
$$

where for each $\left.k \geq 0,\left\{b_{j}^{k} \mid j=1, \ldots, \beta_{k}\right)\right\}$ is a basis for $H_{k}(X)$ with corresponding dual basis $\left\{\bar{b}_{j}^{k} \mid j=1, \ldots, \beta_{k}\right\}$ for $H^{k}(X)$.

The pointed set $\pi_{0}\left(X^{X}, f\right)$ is just the set of homotopy classes of maps $g: X \rightarrow X$ with $f$ representing the distinguished basepoint. For $X$ path connected,

$$
\Lambda_{0}(f ; \mathbb{F})([g])=L(g)\left[x_{0}\right]
$$

where $L(g)$ is the Lefschetz number of $g: X \rightarrow X$ and $\left[x_{0}\right] \in H_{0}(X)$ is represented by any point $x_{0} \in X$ (see Remark 2.2 ).

The invariant $\chi_{1}(X ; \mathbb{F})$ was extensively studied in $[\mathrm{GN}]$ where applications to group theory were given.

Let $h: \pi_{n}\left(X^{X}, f\right) \rightarrow H_{n}\left(X^{X}\right)$ be the composite of the Hurewicz map $\pi_{n}\left(X^{X}, f\right)$ $\rightarrow H_{n}\left(X^{X} ; \mathbb{Z}\right)$ and the coefficient homomorphism $H_{n}\left(X^{X} ; \mathbb{Z}\right) \rightarrow H_{n}\left(X^{X}\right)$. We call $h$ the Hurewicz map over $\mathbb{F}$. Note that $h$ is a homomorphism when $n>0$.

Recall that the evaluation trace $\mathcal{E}: H_{*}\left(X^{X}\right) \rightarrow H_{*}(X)$ was defined to be the Knill trace of the evaluation map $E: X^{X} \times X \rightarrow X$ (see Definition 2.8). Proposition 2.9 implies:

Proposition 5.2. The $n^{\text {th }}$ spherical Lefschetz characteristic is the composite of the Hurewicz map over $\mathbb{F}$ and the evaluation trace, i.e. $\Lambda_{n}(f ; \mathbb{F})=\mathcal{E} \circ h$.

Corollary 5.3. For $n>0, \Lambda_{n}(f ; \mathbb{F}): \pi_{n}\left(X^{X}, f\right) \rightarrow H_{n}(X)$ is a homomorphism. $\square$

The product theorems of $\S 4$ (see Theorems 4.3 and 4.5 ) immediately yield:

Theorem 5.4 (Product Theorem for $\Lambda_{n}$ ). Let $K \stackrel{i}{\rightarrow} X \stackrel{p}{\rightarrow} B$ be an orientable Serre fibration where $B$ is homology equivalent over $\mathbb{F}$ to a finite complex and $H_{*}(K)$ is finite dimensional. Let $\gamma: S^{n} \times X \rightarrow X$ be a fiber map with $\left.\gamma\right|_{\{v\} \times X}=f$ and let $\hat{\gamma} \in \pi_{n}\left(X^{X}, f\right)$ be the associated homotopy element. Let $\hat{\gamma}_{K} \in \pi_{n}\left(K^{K},\left.f\right|_{K}\right)$ be the 
homotopy element corresponding to the restriction, $\left.\gamma\right|_{S^{n} \times K}: S^{n} \times K \rightarrow K$, of $\gamma$ to the fiber. Then, if $\chi(B)$ is the Euler characteristic of $B$,

$$
\Lambda_{n}(f ; \mathbb{F})(\hat{\gamma})=\chi(B) i_{*}\left(\Lambda_{n}\left(\left.f\right|_{K}, \mathbb{F}\right)\left(\hat{\gamma}_{K}\right)\right)
$$

If, in addition, the fibration $p$ is trivial (i.e. $p$ is fiber homotopy equivalent to the projection $K \times B \rightarrow B$ ) then the hypothesis on $B$ can be weakened to the assumption that $H_{*}(B)$ is finite dimensional.

In case $f=\mathrm{id}_{X}$, the conclusion of Theorem 5.4 is:

Corollary 5.5. $\chi_{n}(X ; \mathbb{F})(\hat{\gamma})=\chi(B) i_{*}\left(\chi_{n}(K ; \mathbb{F})\left(\hat{\gamma}_{K}\right)\right)$.

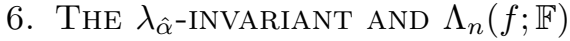

In $[\mathrm{LO}]$, a new homotopy invariant was defined to mimic certain obstructions arising in symplectic geometry. This invariant, denoted by $\lambda_{\hat{\alpha}}$, is closely related to the spherical Lefschetz and Euler characteristic. In this section, we first recall the definition of $\lambda_{\hat{\alpha}}$ as well as several of its important properties, and then show that the spherical Lefschetz characteristic may be written in terms of $\lambda_{\hat{\alpha}}$ (see Propositions 6.8 and 6.9). In particular, we show that $\lambda_{\hat{\alpha}}$ is a homomorphism of negative degree whose trace (as in Definition 1.5) is the spherical Lefschetz characteristic. Using this, we will show in $\S 7$ how the $1^{\text {st }}$ spherical Euler characteristic (also called the first order Euler characteristic in [GN]) may be applied in symplectic geometry.

\section{(A) The $\lambda_{\hat{\alpha}}$-invariant.}

Choose basepoints $v \in S^{n}$ and $x_{0} \in X$. An element of $\pi_{n}\left(X^{X}, f\right)$ is represented by a map $\hat{\alpha}:\left(S^{n}, v\right) \rightarrow\left(X^{X}, f\right)$. We abuse notation by also denoting by $\hat{\alpha}$ the corresponding element in $\pi_{n}\left(X^{X}, f\right)$. By the exponential law (which holds by assumption), there is a map $\alpha: S^{n} \times X \rightarrow X$ given by $\hat{\alpha}(s)(x)=\alpha(s, x)$. Let $\alpha_{\bullet}:\left(S^{n}, v\right) \rightarrow\left(X, f\left(x_{0}\right)\right)$ be defined by $\alpha_{\bullet}(u)=\alpha\left(u, x_{0}\right)$. Then $\operatorname{ev}_{*}(\hat{\alpha})=\alpha \bullet$ where ev: $X^{X} \rightarrow X$ is evaluation at the basepoint. Note that this notation differs from that of [LO], but is consistent with our notation in $\S 2$.

We define a homomorphism $\lambda_{\hat{\alpha}}: H^{q}(X) \rightarrow H^{q-n}(X)$, using the Künneth Formula, by:

$$
\alpha^{*}(y)=1 \times f^{*}(y)+\bar{U} \times \lambda_{\hat{\alpha}}(y),
$$

where $y \in H^{q}(X), \bar{U} \in H^{n}\left(S^{n}\right)$ is the generator such that $\langle\bar{U}, U\rangle=1$ and $U \in$ $H_{n}\left(S^{n}\right)$ is the fundamental homology class (see $\S 5$ ).

Since $\alpha^{*}$ preserves cup products, we easily conclude:

Proposition 6.2. If $n>0$ then $\lambda_{\hat{\alpha}}$ is a derivation of the graded algebra $H^{*}(X)$ of degree - n, i.e. $\lambda_{\hat{\alpha}}(x \cup y)=\lambda_{\hat{\alpha}}(x) \cup y+(-1)^{n|x|} x \cup \lambda_{\hat{\alpha}}(y)$.

In particular, $\lambda_{\hat{\alpha}}$ is a degree $-n$ homomorphism, $H^{*}(X) \rightarrow H^{*}(X)$ and thus has a trace as in Definition 1.5.

Next, we express $\lambda_{\hat{\alpha}}$ in terms of the homomorphism

$$
\bar{\lambda}: H_{*}\left(X^{X}\right) \rightarrow \operatorname{Hom}\left(H^{*}(X), H^{*}(X)\right)
$$


of $\S 2$. There is a commutative diagram:

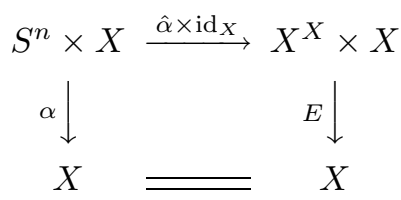

where $E: X^{X} \times X \rightarrow X$ is the evaluation map.

Proposition 6.3. If $n>0$ and $y \in H^{q}(X)$ then

$$
\lambda_{\hat{\alpha}}(y)=h(\hat{\alpha}) \backslash E^{*}(y)=\bar{\lambda}(h(\hat{\alpha}))(y)
$$

where $H_{n}\left(X^{X}\right) \otimes H^{q}\left(X^{X} \times X\right) \stackrel{\backslash}{\rightarrow} H^{q-n}(X)$ is the slant product and $h: \pi_{n}\left(X^{X}, f\right)$ $\rightarrow H_{n}\left(X^{X}\right)$ is the Hurewicz map over $\mathbb{F}$.

Proof. Let $y \in H^{q}(X ; \mathbb{Z})$. By definition, $\bar{\lambda}(h(\hat{\alpha}))(y)=h(\hat{\alpha}) \backslash E^{*}(y)$. Since $\operatorname{deg} U=$ $n>0, U \backslash\left(1 \times f^{*}(y)\right)=0$ and so, by $(6.1)$,

$$
U \backslash \alpha^{*}(y)=U \backslash\left(1 \times f^{*}(y)\right)+U \backslash\left(\bar{U} \times \lambda_{\hat{\alpha}}(y)\right)=0+\langle\bar{U}, U\rangle \lambda_{\hat{\alpha}}(y)=\lambda_{\hat{\alpha}}(y)
$$

and thus:

$$
\lambda_{\hat{\alpha}}(y)=U \backslash \alpha^{*}(y)=U \backslash\left(\hat{\alpha} \times \operatorname{id}_{X}\right)^{*} E^{*}(y)=\hat{\alpha}_{*}(U) \backslash E^{*}(y)=h(\hat{\alpha}) \backslash E^{*}(y) .
$$

Essentially the same calculation in the case $f=\mathrm{id}_{X}$ appears in $\left[\mathrm{Go}_{3},(10.3)\right]$.

One immediate consequence is:

Corollary 6.4. Suppose $n>0$ and $\mathbb{F}$ has characteristic 0 . If $\hat{\alpha} \in \pi_{n}\left(X^{X}, f\right)$ has finite order then $\lambda_{\hat{\alpha}}=0$.

Since $\bar{\lambda}$ is a homomorphism, we have:

Corollary 6.5. For $n>0$, the function $\lambda: \pi_{n}\left(X^{X}, f\right) \rightarrow \operatorname{Hom}\left(H^{*}(X), H^{*-n}(X)\right)$, $\hat{\alpha} \mapsto \lambda_{\hat{\alpha}}$, is a homomorphism.

Consider the special case $f=\operatorname{id}_{X}$ with $\hat{\alpha} \in \pi_{n}\left(X^{X}, \operatorname{id}_{X}\right)$ where $n>0$. Following [Go3], the associated map $\alpha$ or, equivalently, the element $\hat{\alpha} \in \pi_{n}\left(X^{X}, \operatorname{id}_{X}\right)$ may be viewed as a clutching map [Sp, p. 455] along the equator of $S^{n+1}$ which constructs a fibration

$$
X \stackrel{i}{\rightarrow} E \rightarrow S^{n+1}
$$

with $\alpha_{\bullet}=\partial_{\#}(1) \in \operatorname{im}\left(\partial_{\#}: \pi_{n+1} S^{n+1} \rightarrow \pi_{n} X\right)$. Such a fibration has a Wang sequence associated to it:

$$
\cdots \rightarrow H^{q}(E) \stackrel{i^{*}}{\longrightarrow} H^{q}(X) \stackrel{\lambda_{\hat{\alpha}}}{\rightarrow} H^{q-n}(X) \rightarrow H^{q+1}(E) \rightarrow \cdots
$$

and the homomorphism $\lambda_{\hat{\alpha}}$ appearing in this sequence coincides with the $\lambda_{\hat{\alpha}^{-}}$ invariant above. By Proposition 6.2, the homomorphism $\lambda_{\hat{\alpha}}$ is a derivation on $H^{*}(X)$. In the above context, $X \rightarrow E \rightarrow S^{n+1}$ is called the Wang fibration associated to $\hat{\alpha}$ and $\lambda_{\hat{\alpha}}$ is called the Wang derivation.

Another way to think of this is as follows. There is a classifying space $B \operatorname{Aut}(X)$ for fibrations with fiber $X$ which obeys $\pi_{i+1} B \operatorname{Aut}(X)=\pi_{i}\left(X^{X}, \operatorname{id}_{X}\right)$. Therefore, $\hat{\alpha} \in \pi_{n}\left(X^{X}, \mathrm{id}_{X}\right)$ corresponds to an element in $\pi_{n+1} B$ Aut $(X)$ represented by a map $S^{n+1} \rightarrow B \operatorname{Aut}(X)$. The fibration $X \rightarrow E \rightarrow S^{n+1}$ above is simply the pullback via this map of the classifying fibration over $B \operatorname{Aut}(X)$.

Exactness of the Wang sequence gives: 
Proposition 6.6. For $\omega \in H^{q}(X), \lambda_{\hat{\alpha}}(\omega)=0$ if and only if there exists $\bar{\omega} \in H^{q}(E)$ with $i^{*} \bar{\omega}=\omega$.

Of particular interest is the case where $\lambda_{\hat{\alpha}}$ vanishes. Recall that a fibration $K \rightarrow Z \rightarrow B$ is totally non-cohomologous to zero (or TNCZ) over a field $\mathbb{F}$ if any of the following three equivalent conditions holds:

1. The Serre spectral sequence associated to the fibration collapses to the $E_{2^{-}}$ term.

2. $H^{*}(Z) \rightarrow H^{*}(K)$ is surjective (or, equivalently, $H_{*}(K) \rightarrow H_{*}(Z)$ is injective).

3. $H^{*}(Z) \cong H^{*}(K) \otimes H^{*}(B)$ as $H^{*}(B)$-modules.

The equivalence of the first two conditions follows from an examination of the edge homomorphism $H^{*}(Z) \rightarrow H^{*}(K)$ together with the derivation property of differentials in the Serre spectral sequence ([Mc, p. 138]). The third condition is a straightforward modification of the Leray-Hirsch theorem. The TNCZ condition has proven especially important in the subject of transformation groups ([Bo, §XII], $[\mathrm{Br}])$. Indeed, we relate our spherical Euler characteristic to the TNCZ condition by displaying the former as an obstruction to the latter in the case of a compact Lie group action. The exactness of the Wang sequence allows us to formulate a global version of Proposition 6.6: For a Wang fibration $X \stackrel{i}{\rightarrow} E \rightarrow S^{n+1}$, if $H^{*}(E) \rightarrow H^{*}(X)$ is surjective, then $\lambda_{\hat{\alpha}}=0$ identically since it is the "boundary" homomorphism in the Wang sequence. Clearly, the converse holds as well. We record this observation as:

Corollary 6.7. $\lambda_{\hat{\alpha}}=0$ if and only if the Wang fibration associated to $\hat{\alpha}$ is TNCZ. $\square$

(B) Cohomological computation of $\Lambda_{n}(f ; \mathbb{F})$.

We now establish the relationship between the spherical Lefschetz characteristic $\Lambda_{n}(f ; \mathbb{F})$ of $\S 5$ and the $\lambda_{\hat{\alpha}}$-invariant of $\S 6(\mathrm{~A})$.

Let $\hat{\alpha} \in \pi_{n}\left(X^{X}, f\right)$ with associated maps $\alpha: S^{n} \times X \rightarrow X$ and $\alpha_{\bullet}: S^{n} \rightarrow X$.

Proposition 6.8. If $n>0$ then $\Lambda_{n}(f ; \mathbb{F})(\hat{\alpha})=(-1)^{n} L_{n}\left(\lambda_{\hat{\alpha}}\right)$.

Proof. By Proposition 2.11, $\Lambda_{n}(f ; \mathbb{F})(\hat{\alpha}) \equiv L(\alpha)(U)=(-1)^{n} L_{n}(\bar{\lambda}(h(\hat{\alpha})))$ and by Proposition $6.3 \bar{\lambda}(h(\hat{\alpha}))=\lambda_{\hat{\alpha}}$.

Another formula for $\Lambda_{n}(f ; \mathbb{F})$ is given by:

Proposition 6.9. Suppose $X$ is path connected and $n>0$. Then

$$
(-1)^{n} \Lambda_{n}(f ; \mathbb{F})(\hat{\alpha})=L(f) h\left(\alpha_{\bullet}\right)+f_{*}\left(L_{n}\left(\lambda_{\hat{\alpha}}\right)\right) .
$$

Proof. Let $\left\{b_{j}^{k}\right\}$ be a basis for $H_{k}(X)$ with corresponding dual basis $\left\{\bar{b}_{j}^{k}\right\}$ for $H^{k}(X)$. From (6.1), $\alpha^{*}(x)=1 \times f^{*}(x)+\bar{U} \times \lambda_{\hat{\alpha}}(x)$. We have

$$
\begin{aligned}
\alpha^{*}\left(\bar{b}_{j}^{k}\right) \cap U \times b_{j}^{k} & =\left(1 \times f^{*}\left(\bar{b}_{j}^{k}\right)\right) \cap\left(U \times b_{j}^{k}\right)+\bar{U} \times \lambda_{\hat{\alpha}}\left(\bar{b}_{j}^{k}\right) \cap\left(U \times b_{j}^{k}\right) \\
& =U \times f^{*}\left(\bar{b}_{j}^{k}\right) \cap b_{j}^{k}+(-1)^{n((k-n)-k)}(\bar{U} \cap U) \times\left(\lambda_{\hat{\alpha}}\left(\bar{b}_{j}^{k}\right) \cap b_{j}^{k}\right) \\
& =U \times\left\langle f^{*}\left(\bar{b}_{j}^{k}\right), b_{j}^{k}\right\rangle\left[x_{0}\right]+(-1)^{n}[v] \times\left(\lambda_{\hat{\alpha}}\left(\bar{b}_{j}^{k}\right) \cap b_{j}^{k}\right)
\end{aligned}
$$

where $[v] \in H_{0}\left(S^{n}\right)$ and $\left[x_{0}\right] \in H_{0}(X)$ are the classes represented by the basepoints. Now, $\alpha_{*}\left(U \times\left[x_{0}\right]\right)=h\left(\alpha_{\bullet}\right)$ and $\alpha_{*}([v] \times y)=f_{*}(y)$. Thus

$\bar{b}_{j}^{k} \cap \alpha_{*}\left(U \times b_{j}^{k}\right)=\alpha_{*}\left(\alpha^{*}\left(\bar{b}_{j}^{k}\right) \cap U \times b_{j}^{k}\right)=\left\langle f^{*}\left(\bar{b}_{j}^{k}\right), b_{j}^{k}\right\rangle h\left(\alpha_{\bullet}\right)+f_{*}\left((-1)^{n}\left(\lambda_{\hat{\alpha}}\left(\bar{b}_{j}^{k}\right) \cap b_{j}^{k}\right)\right)$. 
Taking an alternating sum over $k$ and invoking Proposition 1.6 yields the conclusion.

Assume $X$ is path connected. Combining Propositions 6.8 and 6.9 , we obtain a result of some interest:

Theorem 6.10. If $n>0$ then $L(f) h\left(\alpha_{\bullet}\right)=\left(\mathrm{id}-f_{*}\right)\left(L_{n}\left(\lambda_{\hat{\alpha}}\right)\right)$.

Corollary 6.11. Let $\hat{\alpha} \in \pi_{n}\left(X^{X}, f\right)$ with evaluation image $\mathrm{ev}_{\#}(\hat{\alpha})=\alpha_{\bullet} \in \pi_{n}(X)$ where $n>0$. If $f_{*}\left(L_{n}\left(\lambda_{\hat{\alpha}}\right)\right)=L_{n}\left(\lambda_{\hat{\alpha}}\right)$, then either $\alpha_{\bullet}$ is in the kernel of the Hurewicz map over $\mathbb{F}$ or $L(f)=0 \bmod p$ where $p \geq 0$ is the characteristic of $\mathbb{F} . \square$

In particular, we obtain:

Corollary 6.12. If $n>0, f_{*}: H_{n}(X) \rightarrow H_{n}(X)$ is the identity and $L(f) \neq 0$ $\bmod p$ then $J_{n}(X, f) \subset \operatorname{ker}(h)$, i.e. the composite

$$
\pi_{n}\left(X^{X}, f\right) \stackrel{\mathrm{ev}_{\#}}{\longrightarrow} \pi_{n}\left(X, f\left(x_{0}\right)\right) \stackrel{h}{\longrightarrow} H_{n}(X)
$$

is zero.

The special case in which $f=\mathrm{id}$, so that $L(f)=\chi(X)$ and $J_{n}(X, f)=G_{n}(X)$, is a result of Gottlieb, $\left[\mathrm{Go}_{2}\right.$, Theorem 4.4].

\section{Some Computations of $\chi_{n}(X ; \mathbb{F})$}

The description of $\chi_{n}(X ; \mathbb{F})(\hat{\alpha})$ in terms of $\lambda_{\hat{\alpha}}$ (Proposition 6.8) allows us to calculate the former in cases where the latter is known.

Example 7.1. By $\left[\mathrm{Go}_{2}\right.$, Theorem 5.4], the Gottlieb groups of spheres (in their dimensions) are: $G_{2 n}\left(S^{2 n}\right)=0$ and $G_{2 n+1}\left(S^{2 n+1}\right)=2 \mathbb{Z} \subset \mathbb{Z}=\pi_{2 n+1}\left(S^{2 n+1}\right)$ except for the cases $G_{1}\left(S^{1}\right)=\mathbb{Z}, G_{3}\left(S^{3}\right)=\mathbb{Z}, G_{7}\left(S^{7}\right)=\mathbb{Z}$. If $\hat{\alpha} \in \pi_{m}\left(S^{m S^{m}}, \operatorname{id}_{S^{m}}\right)$ and $\bar{U} \in H^{m}\left(S^{m} ; \mathbb{F}\right)$ is dual to the fundamental homology class, then (as shown in $[\mathrm{LO}])$,

$$
\lambda_{\hat{\alpha}}(\bar{U})=\left\langle\bar{U}, h\left(\alpha_{\bullet}\right)\right\rangle
$$

where $\alpha_{\bullet}=\operatorname{ev}_{\#}(\hat{\alpha})$ as above. Since $h\left(\alpha_{\bullet}\right)=0$ for all even dimensional spheres,

$$
\chi_{2 n}\left(S^{2 n} ; \mathbb{F}\right)=0 .
$$

For odd dimensional spheres (excluding $S^{1}, S^{3}$ and $S^{7}$ ),

$$
\chi_{2 n+1}\left(S^{2 n+1} ; \mathbb{F}\right)(\hat{\alpha})=-2 U,
$$

where $\alpha_{\bullet}=\operatorname{ev}_{\#}(\hat{\alpha})$ generates $G_{2 n+1}\left(S^{2 n+1}\right)=2 \mathbb{Z}, U \in H_{2 n+1}\left(S^{2 n+1} ; \mathbb{F}\right)$ is the fundamental homology class and $h\left(\alpha_{\bullet}\right)=2 U$. If $2 n+1=1,3,7$, then

$$
\chi_{2 n+1}\left(S^{2 n+1} ; \mathbb{F}\right)(\hat{\alpha})=-U \text {. }
$$

This last calculation generalizes [GN, Example 3.1].

Corollary 5.5 and the above calculation yield: 
Example 7.2. Let $X=S^{n} \times Y$, where $n$ is an odd number. If $n \neq 1,3,7$, then $\chi_{n}(X ; \mathbb{F})(\hat{\alpha})=-\chi(Y) h\left(\alpha_{\bullet}\right)=-2 \chi(Y) U$, where $\alpha_{\bullet}$ generates $G_{n}\left(S^{n}\right)=2 \mathbb{Z}$ and $U \in H_{n}\left(S^{n} ; \mathbb{F}\right)$ is the fundamental homology class. If $n=1,3,7$, then $\chi_{n}(X ; \mathbb{F})(\hat{\alpha})=-\chi(Y) U$, where $\alpha$ • generates $G_{n}\left(S^{n}\right)=\mathbb{Z}$.

Combining Proposition 6.8 (in the case $f=\operatorname{id}_{X}$ ) with Corollary 6.7, we obtain:

Theorem 7.3. Let $\hat{\alpha} \in \pi_{n}\left(X^{X}, \mathrm{id}_{X}\right)$ where $n>0$. If the associated Wang fibration $X \rightarrow E \rightarrow S^{n+1}$ is totally noncohomologous to zero $(T N C Z)$, then $\chi_{n}(X ; \mathbb{F})(\hat{\alpha})=$ 0 .

This observation can be applied to group actions. Suppose $G \times X \rightarrow X$ is an action of a compact Lie group on $X$. Let $S^{n} \rightarrow G$ represent a nontrivial element of $\pi_{n}(G), n>0$, and form the composition $S^{n} \rightarrow G \rightarrow\left(X^{X}, \operatorname{id}_{X}\right)$. Denote the class of this map by $\hat{\alpha} \in \pi_{n}\left(X^{X}, \operatorname{id}_{X}\right)$. Now, $\pi_{n}(G) \cong \pi_{n+1}(B G)$, so we obtain a pullback of the Borel fibration $X \stackrel{j}{\rightarrow} E G \times_{G} X \rightarrow B G$ associated to the action,

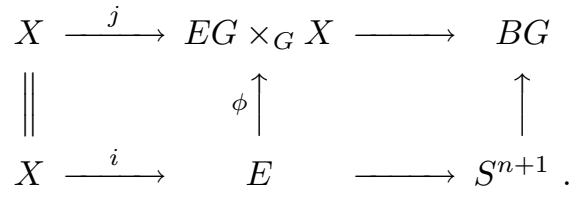

The fibration $X \stackrel{i}{\rightarrow} E \rightarrow S^{n+1}$ is precisely that associated to $\hat{\alpha}$, so the associated Wang derivation is $\lambda_{\hat{\alpha}}$. By the commutativity of the diagram, if the Borel fibration is TNCZ, then so is the Wang fibration. From Theorem 7.3 we then obtain:

Corollary 7.4. If $n>0$ and the Borel fibration of the action is TNCZ, then $\chi_{n}(X ; \mathbb{F})(\hat{\alpha})=0$.

A particularly important case where a group action has a TNCZ Borel fibration (over $\mathbb{Q}$ ) is when $X$ is a closed symplectic manifold with a Hamiltonian circle action (see [Kir]). Recall that $X^{2 n}$ is symplectic if there is a closed 2-form $\omega$ on $X$ with the property that the wedge product $\omega^{n}$ is a volume form for $X$. An $S^{1}$-action on $X$ is Hamiltonian if the 1 -form $i_{Y} \omega$ is exact where $Y$ is the fundamental vector field associated to the action and $i_{Y} \omega$ is defined by $i_{Y} \omega(Z)=\omega(Y, Z)$. Thus,

Corollary 7.5. If $X$ is a closed symplectic manifold and $\hat{\alpha} \in \pi_{1}\left(X^{X}, \mathrm{id}_{X}\right)$ corresponds to a Hamiltonian $S^{1}$-action, then $\chi_{1}(X ; \mathbb{Q})(\hat{\alpha})=0 .{ }^{3}$

In [LO] it was shown that the 1 -form $i_{Y} \omega$ is exact if and only if $\lambda_{\hat{\alpha}}(\omega)=0$ (where we use $\omega$ here to denote the cohomology class). In fact, any $S^{1}$-action on a cohomologically symplectic manifold $X$ (i.e. $X$ is a closed $2 n$-dimensional manifold with a class $\omega \in H^{2}(X ; \mathbb{Q})$ such that $\left.\omega^{n} \neq 0\right)$ is said to be $c$-Hamiltonian if $\lambda_{\hat{\alpha}}(\omega)=$ 0 . It was also shown in $[\mathrm{LO}]$ that the condition $\lambda_{\hat{\alpha}}(\omega)=0$ implies that $\left.\lambda_{\hat{\alpha}}\right|_{H^{1}(X ; \mathbb{Q})}=$ 0 . Indeed, these two conditions are equivalent if $X$ is "homologically Kähler". Recall that $X^{2 n}$ is homologically Kähler if it is cohomologically symplectic and $\omega \in H^{2}(X ; \mathbb{Q})$ has the "Hard Lefschetz Property": for $j \leq n$ the homomorphism $H^{j}(X ; \mathbb{Q}) \rightarrow H^{2 n-j}(X ; \mathbb{Q})$ given by multiplication by $\omega^{n-j}$ is an isomorphism. For homologically Kähler spaces, it is known (see [B]) that any cohomology derivation of negative degree which is zero on $H^{1}(X ; \mathbb{Q})$ is identically zero. By Propositions 6.2 and 6.8 ,

\footnotetext{
${ }^{3}$ See note added in proof.
} 
Proposition 7.6. Suppose $X$ is homologically Kähler. If $\hat{\alpha} \in \pi_{1}\left(X^{X}, \mathrm{id}_{X}\right)$ is cHamiltonian $\left(\right.$ i.e. $\left.\lambda_{\hat{\alpha}}(\omega)=0\right)$, then $\chi_{1}(X ; \mathbb{Q})(\hat{\alpha})=0$. In particular, if an $S^{1}$-action on $X$ (with associated $\hat{\alpha})$ is $c$-Hamiltonian, then $\chi_{1}(X ; \mathbb{Q})(\hat{\alpha})=0$.

If $X$ is simply connected and homologically Kähler then $H^{1}(X ; \mathbb{Q})=0$ and so any derivation of $H^{*}(X ; \mathbb{Q})$ of negative degree is trivial. By Propositions 6.2 and 6.8 ,

Proposition 7.7. If $X$ is homologically Kähler and simply connected then

$$
\chi_{n}(X ; \mathbb{Q})=0 \text { for all } n \geq 1 .
$$

Example 7.8. Let $X=\mathbb{C} P^{n}$. The complex manifold $\mathbb{C} P^{n}$ is Kähler and simply connected. By Proposition $7.7, \chi_{n}\left(\mathbb{C} P^{n} ; \mathbb{Q}\right)=0$ for all $n \geq 1$.

From these results, we see that the spherical Euler characteristics may be thought of as obstructions to homotopy properties such as the TNCZ condition, and to a manifold being homologically Kähler.

Added in proof. In a forthcoming paper and using different methods the first two authors have obtained the conclusion of Corollary 7.5 for any smooth circle action on any even-dimensional closed manifold. This also holds with integer coefficients.

\section{REFERENCES}

[BG] J. C. Becker and D. H. Gottlieb, Transfer maps for fibrations and duality, Compositio Math. 33 (1976), 107-133. MR 55:9087

[B] A. Blanchard, Sur les variétés analytiques complexes, Annales Ec. Norm. Sup. 73 (1956), 157-202. MR 19:316a

[Bo] A. Borel, Seminar on Transformation Groups, Princeton U. Press, Princeton, NJ, 1960. MR 22:7129

[Br] G. Bredon, Introduction to Compact Transformation Groups, Academic Press, New York, 1972. MR 54:1265

$\left[\mathrm{D}_{1}\right]$ A. Dold, Fixed point index and fixed point theorem for Euclidean neighborhood retracts, Topology 4 (1965), 1-8. MR 33:1850

$\left[\mathrm{D}_{2}\right] \quad$ A. Dold, The fixed point transfer of fibre-preserving maps, Math. Z. 148 (1976), 215-244. MR 55:6416

$\left[\mathrm{D}_{3}\right]$ A. Dold, Lectures on algebraic topology, Springer-Verlag, New York, 1980. MR 82c:55001

[GN] R. Geoghegan and A. Nicas, Higher Euler characteristics (I), L'Enseign. Math. 41 (1995), 3-62. CMP 95:15

[Go1] D. Gottlieb, A Certain Subgroup of the Fundamental Group, Amer. J. Math. 87 (1965), 840-856. MR 32:6454

$\left[\mathrm{Go}_{2}\right]$ D. Gottlieb, Evaluation Subgroups of Homotopy Groups, Amer. J. Math. 91 (1969), 729 756. MR 43:1181

[Go3] D. Gottlieb, Applications of Bundle Map Theory, Trans. Amer. Math. Soc. 171 (1972), 23-50. MR 46:8222

[Kir] F. C. Kirwan, Cohomology of Quotients in Symplectic and Algebraic Geometry, Math. Notes Vol. 31, Princeton Univ. Press, Princeton, 1984. MR 86i:58050

[Kn] R. J. Knill, On the homology of a fixed point set, Bull. Amer. Math. Soc. 77 (1971), 184-190. MR 45:9320

[Le] J. Leray, Sur les équations et les transformations, J. Math. Pures Appl. (9) 24 (1946), 201-248. MR 7:468g

[LO] G. Lupton and J. Oprea, Cohomologically symplectic spaces: toral actions and the Gottlieb group, Trans. Amer. Math. Soc. 347 (1995), 261-288. MR 95f:57056

[M] S. Mac Lane, Homology, Van Nostrand, Princeton, NJ, 1967. MR 50:2285 
[Mc] J. McCleary, User's Guide to Spectral Sequences, Publish or Perish Inc., Wilmington, Del., 1985. MR 87f:55014

[Sp] E. H. Spanier, Algebraic topology, McGraw-Hill, New York, 1966. MR 35:1007

[W] G. W. Whitehead, Elements of Homotopy Theory, Springer-Verlag, New York, 1978. MR 80b:55001

Department of Mathematics, SUny at Binghamton, Binghamton, New York 13902 6000

E-mail address: ross@math.binghamton.edu

Department of Mathematics, McMaster University, Hamilton, Ontario L8S 4K1, CANAdA

E-mail address: nicas@mcmaster.ca

Department of Mathematics, Cleveland State University, Cleveland, Ohio 44115

E-mail address: oprea@math.csuohio.edu 